\title{
Modelling Surtseyan Ejecta
}

\author{
Emma Elizabeth Greenbank
}

Supervisor: Professor Mark McGuinness

\section{VICTORIA UNIVERSITY OF WELLINGTON \\ Te Whare Wānanga o te Ūpoko o te Ika a Māui}

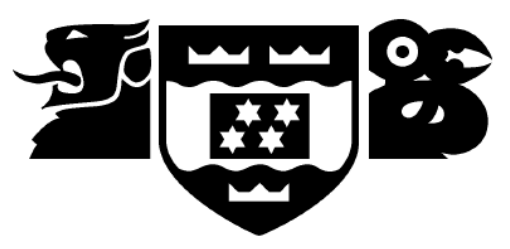

School of Mathematics, Statistics and Operations Research

Te Kura Mātai Tatauranga, Rangahau Pūnaha

\author{
A thesis \\ submitted to the Victoria University of Wellington \\ in fulfilment of the requirements for the degree of \\ Master of Science \\ in Mathematics.
}

Victoria University of Wellington

2015 



\begin{abstract}
Surtseyan ejecta are formed in shallow sub-aqueous volcanic eruptions. They occur when water, containing a slurry of previously erupted material, is washed into the volcanic vent. This slurry is incorporated into the magma and ejected from the volcano inside a ball of magma. These magma bombs containing entrained material are called, Surtseyan ejecta or Surtseyan bombs .

At the time of entrainment there is a large temperature difference between the magma (at approximately $1000^{\circ} \mathrm{C}$ ) and the slurry (at approximately $20^{\circ} \mathrm{C}$ ). As the inclusion temperature increases, the water contained in the slurry evaporates, causing an increase in the pressure at the boundary of the entrainment. This pressure increase is offset by the vapour diffusing through the pores of the magma. If the pressure exceeds the tensile strength of the surrounding magma the Surtseyan ejecta will rupture.

The volcanological question of interest is whether the magma ruptures. There is evidence of intact ejecta so it can be concluded that rupture does not always occur. We have developed a set of equations that transiently model the changes in temperature and pressure in Surtseyan ejecta. Numerical solutions show that the pressure rapidly increases to a stable value. Because the pressure reaches equilibrium a steady-state solution can be used to determine the maximum pressure and a criterion for rupture.
\end{abstract}





\section{Acknowledgements}

Firstly, I owe a debt of gratitude to my supervisor Professor Mark McGuinness. At all times his door was open and no matter how trivial a problem he was always willing to support and guide me. This also included the opportunity to attend conferences so I could share ideas with others in the field. Without his dedicated and patient help I doubt I would have reached this point.

I also would like to thank Dr Ian Schipper for this fascinating problem and for his insight into Surtseyan ejecta behaviour.

My gratitude should also go to Dr Dimitrios Mitsotakis for agreeing to be my second supervisor and the School of Mathematics, Statistics and Operations Research, particularly Dr Steven Archer, for providing me with tutoring work throughout my masters. I also wish to acknowledge my fellow graduate students for their encouragement and for their emotional support.

The NZMS, ANZIAM and Victoria University provided me with grants that allowed me to attend conferences and workshops during my masters year and for this I am grateful.

Finally, I want to thank my parents Christine and Ian, and my brothers William and Richard for their love and support throughout all my studies. 



\section{Contents}

1 Introduction 1

1.1 Background $\ldots \ldots \ldots \ldots \ldots \ldots$

1.2 Literature Review . . . . . . . . . . . . . . . . . . . 3

\begin{tabular}{|lll}
\hline 2 & The Transient Model & 13
\end{tabular}

$2.1 \quad$ Confined Vapour Model . . . . . . . . . . . . . . . . . . . . . . . 14

2.2 Biot Number . . . . . . . . . . . . . . . . . . 15

2.3 The Thermal equation . . . . . . . . . . . . . . . . . 17

2.4 Amount of heat required to reach equilibrium . . . . . . . . . . . 19

2.5 Estimating the temperature gradient . . . . . . . . . . 21

2.6 Pressure Equations $\ldots \ldots \ldots \ldots . \ldots \ldots$

2.7 Non-Dimensionalisation . . . . . . . . . . . . . . . . . . . . . . 29

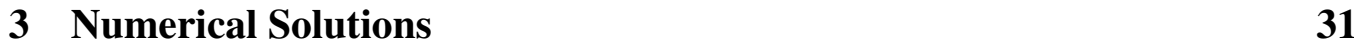

$3.1 \quad$ Results . . . . . . . . . . . . . . . . . . . . 34

4 Conclusion and Discussion 4

$4.1 \quad$ Discussion . . . . . . . . . . . . . . . . . . . 41

4.2 Conclusion . . . . . . . . . . . . . . . . . 43

\begin{tabular}{|ll}
\hline A Symbol Glossary & 45
\end{tabular} 
\begin{tabular}{|lr}
\hline P Programming Numerics in Matlab & 53
\end{tabular}

\begin{tabular}{lr}
\hline Bibliography & 55
\end{tabular} 


\section{Chapter 1}

\section{Introduction}

\subsection{Background}

The island of Surtsey was created by a volcanic eruption in Vestmanyaar, off the coast of Iceland, on the 14th of November 1963. This eruption remains the archetype for this class of marine shallow to emergent explosive volcanic eruptions known as Surtseyan eruptions. Other examples of these eruptions include the 16th January 2015 eruption in Tonga and some similar features can be seen in past Ruapehu (NZ) eruptions.

Surtseyan eruptions occur when a volcanic vent is situated at the surface of a body of water. The mixing of the magma and water causes the violent explosive activity that is a characteristic of Surtseyan eruptions [13]. During the eruption water containing previously erupted material is washed back into the volcanic vent and incorporated into the magma. There is evidence that one outcome is that entrained slurry is ejected, from the volcano, inside balls of magma called Surtseyan ejecta. The mechanism of the creation of Surtseyan ejecta is still not well understood.

At the time of entrainment Surtseyan ejecta have a large temperature difference between the magma (at approximately $1000^{\circ} \mathrm{C}$ ) and the inclusion $\left(20^{\circ} \mathrm{C}\right)$. This temperature difference causes the water in the entrained slurry to rapidly evaporate. The surrounding magma does not significantly expand resulting in the pressure at the boundary of inclusion to increase until the water is depleted. This 


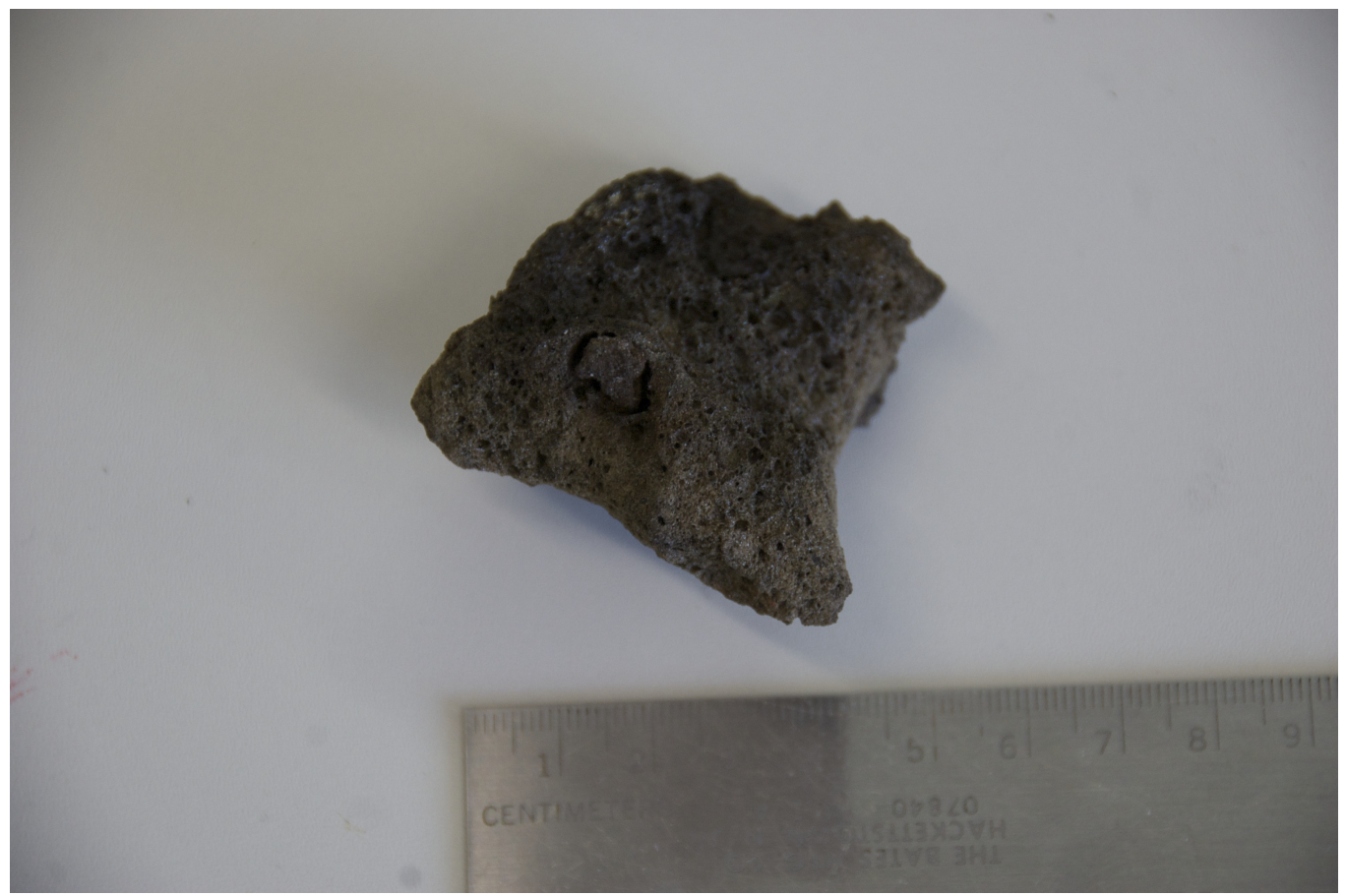

Figure 1.1: Photo of a Surtseyan ejecta taken by Mark McGuinness. An inclusion is visible just left of the centre, with a void space around it.

pressure increase is offset by the water vapour diffusing through the surrounding magma. If the pressure exceeds the tensile strength of the magma, the critical pressure, the Surtseyan ejecta will rupture. Intact Surtseyan ejecta have been found on Surtsey and it can therefore be concluded that this critical pressure is not always reached. Figure 1.1 is a photo of a small intact Surtseyan ejecta, from the eruption at Surtsey. Surtseyan ejecta come in a variety of sizes and they are categorized in three groups:

- $\operatorname{Ash}(<2 \mathrm{~mm}$ in diameter $)$

- Lapilli $(\approx 2-64 \mathrm{~mm}$ in diameter $)$

- $\operatorname{Bomb}(\geq 64 \mathrm{~mm}$ in diameter $)$

Lapilli and bombs often contain multiple inclusions, of entrained ash, lapilli and water. After a Surtseyan ejecta is cooled there exists a void space around the inclusion. This is due to the compacting of the sediments as the water evaporates. This void can be seen surrounding the inclusion in Figure 1.1. 
The volcanological question of interest is whether the ejecta ruptures before the water source is depleted. If the vapour is confined to the inclusion, pressures rise and exceed the tensile strength of magma by approximately a factor of 300 (see Section 2.1). This would indicate that ejecta would always explode. Evidence of intact ejecta has been found which would suggest that sometimes the pressures do not reach the levels seen in Section 2.1. It can be concluded that the gas escaping though the pores is not negligible and needs to be included in the model.

The following chapters show the development of a set of equations that model Surtseyan ejecta behaviour and the conditions needed for rupture. These equations were solved numerically and the results are be used to provide Ian Schipper, a geologist from Victoria University of Wellington, with a criterion for the rupture of Surtseyan ejecta to use in his studies of this phenomenon.

\subsection{Literature Review}

The modelling of Surtseyan ejecta is a new area in mathematical modelling. There is currently no literature that provides a mathematical model for the behaviour of Surtseyan ejecta. The available literature concerns the mechanisms of Surtseyan volcanism and the mixing of water and magma.

\subsubsection{Surtseyan Eurptions}

The addition of water in the subaqueous eruption process produces hazards that are not normally seen in dry land volcanoes. Mastin and Witter [16] show that subaqueous eruptions make up $8 \%$ of the world's eruptions but cause $20 \%$ of the fatalities associated with volcanic eruptions. The high fatality rate is due to lahars, tsunami and base surges that have the potential to cause devastation far away from the eruption. Base surges are caused by the mixing of water and magma producing fast moving clouds of gas and ash. There are two mechanisms that drive the explosive mixing of magma and water in central vent eruptions. Mastin et al. [17] describe the two mechanisms as:

1. the lowering of magma in a channel below the water table followed by an influx of water 
2. the jetting of magma through surface water or through a wet crater that has water entering though the porous walls.

The first mechanism occurred in Ukinrek Maars eruption in Alaska in 1977 and the second is the mechanism driving the eruption at Surtsey.

Surtseyan volcanism is characterised by intermittent, almost silent, jets or a continuous uprush of tephra along with a large amount of steam. This behaviour has been observed to persist as long as the water can flood across or through the top of the volcanic pile into the vent. Kokelaar's model for Surtseyan eruption processes is an explanation for the observed characteristics ([13] [14]). He proposes that there exists a funnel shaped vent filled with a slurry of water and previous erupted material. This is surrounded by a ring of previous erupted material which is slipping back into the vent. As the volcano erupts the slurry is continuously incorporated into the magma and then is replenished as more slurry is washed from the pile into the vent. Pockets of liquid water enclosed in the magma cause violent and continuous expansion producing the characteristic jets seen in Surtseyan eruptions. If there is a higher rate of magma supply a continuous uprush of tephra is the result instead of the intermittent jets. When the water can no longer be replenished the slurry, in the vent, gradually dries and the eruptive style changes to Hawaiian fountaining of incandescent magma.

Fragmentation and magma mixing processes are difficult to observe directly. In order to quantitatively determine the mechanisms causing magma mixing and fragmentation, a textural study of the pyroclasts is needed. Zimanowski et al. [31] studies the differences in the pyroclasts between those produced by decompression or molten fuel coolant interaction. A textural study of Surtseyan ejecta could identify features that would indicate whether ejecta are produced by the mingling of magma and water.

\subsubsection{Magma Fragmentation}

There currently exists no mathematical modelling for the fragmentation of the magma in Surtseyan ejecta. There are studies concerning the fragmentation of magma in high energy eruptions ([10] [1] [24] ) and some of these concepts could be applied to the Surtseyan ejecta model. High energy volcanic eruptions 
(Volcanian, Plinian and Ultra Plinian) are the result of a build up of pressure that undergoes rapid decompression. This depressurization can be caused by either the collapsing of a dome or dislodging of a plug in the volcanic vent. The sudden pressure change causes fragmentation of the magma. This is similar to the build up of pressure in Surtseyan ejecta causing the magma to rupture.

Magma fragmentation is difficult to study as it occurs inside the volcanic vent. To study this process accurately an experiment using an analogue material is required. Alidibirov [1] designed a vertical shock tube apparatus that is used to investigate the mechanisms driving magma fragmentation. This experiment has been modified to solve a variety of different problems. For example Spieler [27] research on the fragmentation threshold of pyroclastic rocks. The experiment takes a sample of porous volcanic rock and uses it as an analogue material for magma. This analogue material for magma is justified because in the time-scale of the experiment the magma would act like a brittle solid. This rock is glued into a sample holder. This sample is placed inside an autoclave which is attached to a larger chamber at atmospheric pressure. These two chambers are separated by a diaphragm. The autoclave is slowly pressurized and rapid decompression is triggered by a failure in the diaphragm. A high speed video camera is used to record the results.

There have been proposed many different fragmentation mechanisms. These mechanisms can be categorised into groups depending on the viscosity and temperature of the magma. A proposed mechanism for magma fragmentation at a low viscosity is bubble formation. This was first suggested by Verhoogen [29] and was later modified by McBirney and Murase [19]. Bubbles are formed in the magma after decompression from the gases enclosed in the porous magma. When the magma is depressurised the enclosed gas has a higher pressure than the surface. This pressure difference deforms the low viscosity magma and expands the pores to form bubbles. If the volume ratio of bubbles to magma becomes too large fragmentation of the magma occurs. Sparks proposed explosive fragmentation is the result of these bubbles bursting [25].

If the magma has a higher viscosity, then a larger force will be required to deform it and the gases trapped in the pores cannot expand to reduce the pressure. This causes an over pressure in the vesicles. There is debate over the mechanism 
causing fragmentation in this case. Bennet [5] proposed that the mechanism relies on expansion waves and that it can be studied using one dimensional shock tube experiments. Another theory (Sparks [25]) argues that the bubbles in the magma would create their own expansion and compression waves and every bubble would act as its own diaphragm. There is also a theory that fragmentation depends on turbulence more than the fluidization processes (Valentine [28]).

A mathematical model for explosive fragmentation of vesicular magma is presented by Fowler et al. [10]. This model considers the flow of vapour though a sample of porous rock in Alidibirov's [1] experimental set up. The fragmentation mechanism is controlled by the gas pore pressure exceeding the yield stress, the lowest stress needed for the rock to rupture, of the porous rock. At the time of rapid decompression the decreasing pressure at the surface causes a over pressure in the pores of the rock. This creates a pressure difference between the pores and the surface that will cause fragmentation if it exceeds the yield stress.

The model consists of conservation of mass and momentum equations for both the pressurized gas and the solid. It uses a rock base and further work by Singh [24] modified this model to create a model for dusts as they have significantly different tensile strength to rock. In the rock model, taken from Biot's equations, the solid strain tensor is

$$
e_{i j}=\frac{1}{2}\left(\frac{\partial w_{i}}{\partial x_{j}}+\frac{\partial w_{j}}{\partial x_{i}}\right)
$$

where $w$ is the solid displacement. The dilatations are defined by

$$
\begin{aligned}
e & =e_{k k}=\nabla \cdot w, \\
\varepsilon_{f} & =\nabla \cdot W .
\end{aligned}
$$

The dilatation of the solid (Equation (1.2) ) depends of the solid displacement, $w$, and the fluid (Equation (1.3) ) on the gas displacement, $W$. The resulting equations for the stress are

$$
\begin{aligned}
(1-\phi) \sigma_{i j}^{S} & =2 N_{E} e_{i j}+\left[A_{E} e+Q_{E} \varepsilon_{f}\right] \delta_{i j} \\
-\phi p_{g} & =Q_{E} e+R_{E} \varepsilon_{f} \\
(1-\phi) \sigma_{i j}^{S} & =2 N_{E} e_{i j}+\left[B_{1} e-\alpha_{1} p_{g}\right] \delta_{i j} \\
B_{1} & =A_{E}-\frac{Q_{E}^{2}}{R_{E}}
\end{aligned}
$$




$$
\alpha_{1}=\frac{\phi Q_{E}}{R_{E}}
$$

where $p$ is the gas pressure, $\sigma_{i j}^{S}$ is the yield stress, $A_{E}$ and $N_{E}$ are Lamé constants for the solid equation and both $Q_{E}$ and $R_{E}$ are related to the deformability of the pore space and fluid. In Equation (1.6) the stress equations have been rewritten to cancel $\varepsilon_{f}$.

The conservation of fluid momentum equation for a pore fluid is

$$
\rho_{f} \phi v_{t}=-\phi \nabla p_{g}-A_{m}-D_{d}
$$

and for the solid

$$
\rho_{s}(1-\phi)\left(w_{e}\right)_{t t}=(1-\phi) \nabla \cdot \sigma^{S}+A_{m}+D_{d}
$$

where $\rho_{s}$ and $\rho_{f}$ denote the densities of the solid and the fluid respectively, $v$ is the gas velocity, $w_{e}$ is the solid displacement from equilibrium and the subscripts $t$ denote the time derivatives. $A$ is the added mass effect, this represents the beads movement in the fluid, and is taken to be

$$
A_{m}=\rho_{a} \frac{\partial}{\partial t}\left(v-\left(w_{e}\right)_{t}\right) .
$$

$D_{d}$ is the interfacial drag and $b$ is the interfacial drag coefficient. The interfacial drag coefficient is obtained using Darcy's law, with the small acceleration terms ignored. $D_{d}$ and $b$ are defined as

$$
\begin{aligned}
D_{d} & =b\left(v-\left(w_{e}\right)_{t}\right), \\
b & =\frac{n_{f} \phi^{2}}{k}
\end{aligned}
$$

where $n_{f}$ is the gas viscosity and $k$ the gas permeability.

To complete the model the conservation of mass equations

$$
\left(\rho_{f} \phi\right)_{t}+\nabla \cdot\left(\rho_{f} \phi v\right)=0,
$$


for the fluid, and

$$
\left((1-\phi) \rho_{s}\right)_{t}+\nabla \cdot\left((1-\phi) \rho_{s}\left(w_{e}\right)_{t}\right)=0
$$

for the solid along with the state equation for an ideal gas must be considered. After non-dimensionalisation and setting small terms to zero, the gas pressure satisfies

$$
\frac{\partial p_{g}^{\frac{1}{\gamma}}}{\partial t}=\frac{\partial}{\partial z}\left(\left|\frac{p_{g}{ }^{\frac{1}{\gamma}}}{\left(p_{g}\right)_{z}}\right|^{\frac{1}{2}} \frac{\partial p_{g}}{\partial z}\right)
$$

where the subscript $z$ denotes that space derivative. Equation (1.15) is a non-linear diffusion equation in $p_{g}$.

The magma fragmentation model proposed by Fowler et al. [10] considers a porous solid with over-pressurized gas in its pores and the amount of stress required for the solid to fragment. This is similar to the Surtseyan ejecta problem as it also considers an overpressure between the entrained slurry and the magma's surface and when this will cause ejecta to fragment. Therefore some of the methods used in this model, particularly the momentum and stress equations, could be used to solve the Surtseyan ejecta problem. The pressure build-up inside Surtseyan ejecta is driven by a temperature difference, not a rapid decompression, and therefore the temperature analysis needs to be considered.

\subsubsection{Fluid flow in a porous medium}

A simpler approach to that described above, is to consider the equations for fluid flow through a uniformly constant porous media. The equations describing fluid flow in a porous medium were developed in the petroleum, groundwater and soil science literature and were applied to geothermal reservoirs by Grant et al. [11]. In geothermal systems fluid can exist in either liquid, vapour or a mixture of both states in the pores of the medium.

Conservation equations apply separately to each phase, but they can be added together to obtain a single equation for the two phase flow. The mass conservation equation for a single phase flow is

$$
\phi \frac{\partial \rho_{x}}{\partial t}+\nabla \cdot\left(\mathbf{u}_{x}\right)=0
$$


where $\mathbf{u}_{x}$ is the mass flux density and $\rho_{x}$ is the density of the fluid.

The conservation of mass equation for a two phase flow is a combination of the single phase vapour and liquid flows. If the porous media is saturated with a mixture of liquid and vapour, a fraction of the pore space is filled with each phase. The fraction of the pore space containing liquid is denoted $S_{l}$ and as the pore space is saturated with fluid the fraction containing vapour is $1-S_{l}=S_{v}$. The conservation of mass equation for a two phase flow is

$$
\phi \frac{\partial\left(\rho_{l} S_{l}+\rho_{v} S_{v}\right)}{\partial t}+\nabla \cdot\left(\mathbf{u}_{v}+\mathbf{u}_{l}\right)=0
$$

The conservation of energy can also be considered for both single and two-phase fluid flows. Energy can be transferred between the rock and fluid through conduction as well as being carried by the fluid. The single phase conservation of energy equation is given by

$$
\frac{\partial}{\partial t}\left((1-\phi) \rho_{r} U_{r}+\phi \rho U\right)+\nabla \cdot(\mathbf{u H}-K \nabla T)=0
$$

where $U_{r}$ and $U$ are the specific internal energies of the rock and fluid respectively. The energy flux density carried by the fluid is $\mathbf{u H}$, where $H$ is the specific enthalpy of the fluid, the conductive heat flux density is $K \nabla T$ and where $K$ is the conductivity of the rock. The conservation of energy equation for a two phase flow is

$$
\frac{\partial}{\partial t}\left((1-\phi) \rho_{r} U_{r}+\phi\left(S_{l} \rho_{l} U_{l}+S_{v} \rho_{v} U_{v}\right)\right)+\nabla \cdot\left(\mathbf{u}_{v} \mathbf{H}_{v}+\mathbf{u}_{l} \mathbf{H}_{l}-K \nabla T\right)=0 .
$$

In the Surtseyan ejecta problem these equations describe the flow of fluid through the ball of porous magma. The energy conservation equation could be used to calculate the amount of heat escaping the ejecta with the vapour. 


\subsubsection{Injection of geothermal waste model}

This technique described in Chapter 1.2.3 has been used by Pruess [22] to model the injection of geothermal waste (water) back into depleted geothermal reservoirs that contain superheated vapour. The injection of waste water causes a cold front to propagate outwards from the injection site. It is assumed, because of the large temperature difference, that the water flashes to steam over a finite distance allowing the single phase vapour flow equations to be used.

Pruess's model [22] starts with the conservation of mass equation for a single phase vapour flow

$$
\frac{\partial\left(\phi \rho_{v}\right)}{\partial t}=-\nabla \cdot \mathbf{F}_{v}
$$

where $\mathbf{F}_{v}$ is the mass flux that can be calculated using Darcy's law

$$
\mathbf{F}_{v}=-k \frac{\rho_{v}}{\mu_{v}} \nabla p
$$

Darcy's law describes the fluid flow through a porous medium, and in this case the hot vapour though porous rock. Equations (1.20) and (1.21) are combined to form

$$
\frac{\partial\left(\phi \rho_{v}\right)}{\partial t}=-\nabla \cdot\left(-k \frac{\rho_{v}}{\mu_{v}} \nabla p\right)
$$

which is a non-linear vapour density diffusion equation. The vapour density depends on pressure and the ideal gas law can be used to form the non-linear pressure diffusion equation

$$
\frac{\phi}{p} \frac{\partial\left(p^{2}\right)}{\partial t}=\frac{k}{\mu_{v}} \nabla^{2} p^{2}
$$

Equation (1.23) can be simplified to

$$
\frac{\partial\left(p^{2}\right)}{\partial t}=\frac{k \bar{p}}{\mu_{\nu} \phi} \nabla^{2} p^{2}
$$

a linear diffusion equation in $p^{2}$ by assuming an average pressure, $\bar{p}$, in the diffusivity constant. This is a reasonable assumption because the pressure changes are small in the geothermal reservoirs. The equivalent assumption cannot be made in the context of Surtseyan ejecta as there may be large pressure variations. 
In Pruess's model [22] the initial condition is

$$
p^{2}=p_{i}^{2}
$$

where $p_{i}$ is the initial pressure. There are two boundary conditions. At infinity the boundary condition is

$$
\left.\frac{d p^{2}}{d r}\right|_{r \rightarrow \infty}=0
$$

The boundary condition at the front is

$$
\left.\frac{d p^{2}}{d r}\right|_{r=f}=-\frac{2 Z \mu_{v} R T_{0}}{M k} \frac{q_{v f}}{2 \pi r_{f} H}
$$

where $Z$ is the real gas compressibility factor, $T_{0}$ is the initial water temperature, $M$ is the molecular mass of water, $R$ is the gas constant, $k$ is the permeability of the rock, $q_{v f}$ is the mass flow rate at the front and $r_{f}$ is the distance for the injection site to the front. This can be solved analytically, as the diffusivity parameter is known, to give

$$
p^{2}=p_{i}^{2}-\frac{Z R T_{0} \mu_{v}}{2 \pi k H M} q_{v f} e^{\frac{r_{f}^{2} \phi \mu_{v}}{4 \bar{p} k}} E i\left(\frac{-r^{2} \phi \mu_{v}}{4 \bar{p} k}\right)
$$

an approximate solution for the pressure. This approximate solution was confirmed to be reasonable by Pruess [22] using numerical solutions.

Equation 1.23 is used in Chapter 2 as the diffusion equation describing the pressure behaviour of Surtseyan ejecta. The boundary conditions at the front in Pruess's model [22] cannot be used in the Surtseyan ejecta problem as the entrainment only has a finite supply of water. Furthermore, Pruess's model has an expanding front where our model requires a collapsing front. 


\section{Chapter 2}

\section{The Transient Model}

The interior pressure of a Surtseyan ejecta depends on the rate of steam generation, due to the heating of the enclosed slurry, and on the flow of vapour escaping through the pores of the surrounding vesicular magma. Our aim is to create a model that allows us to calculate the transient pressure response from steam generated when slurry is entrained in a larger ball of molten magma. The focus of this study is the magnitude of the maximum pressure produced by the heating of the liquid inside the entrained slurry. The maximum pressure is used to determine whether the ejecta will explode due to the buildup of pressure.

We assume that a single deposit of entrained slurry is placed at the centre of the ball of hot magma, see Figure 2.1. This allows spherical symmetry to be assumed. The magma surrounding the inclusion is a highly viscous liquid which is freezing. This magma is charged with vapour bubbles, called vesicles, due to the water separating from the magma solution; this provides the porosity. The speed with which the pressure changes allows us to treat the viscous liquid as a solid porous medium with cohesive strength [1].

This model will consist of coupled pressure and temperature equations. The temperature equation is a conduction equation considering the conduction of heat from the hot magma to the entrained slurry. The thermal equation is then used to calculate the amount of vapour generated in the entrained slurry. It is this vapour production that drives the pressure change and therefore the vapour flow through the pores of the magma. 


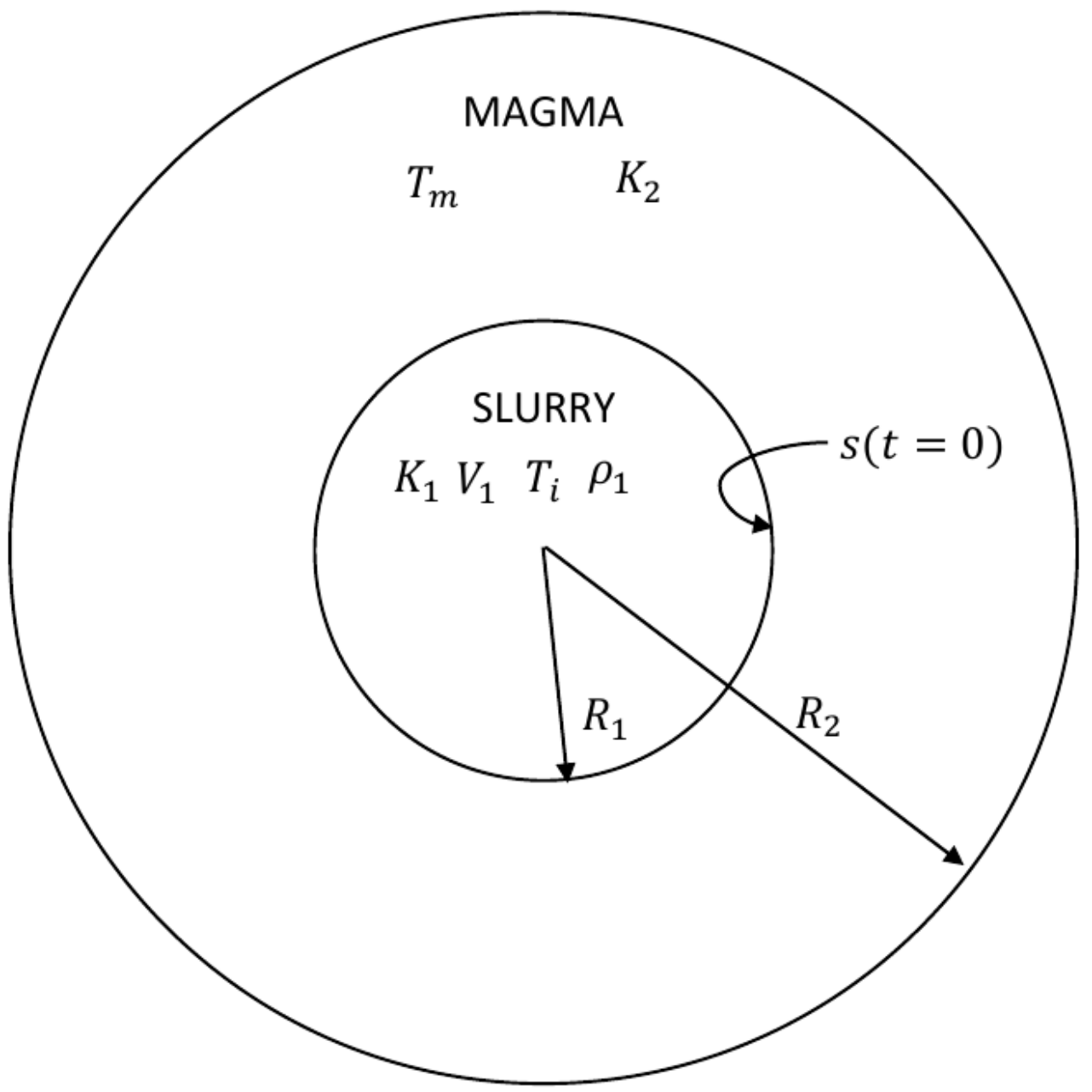

Figure 2.1: Surtseyan ejecta simple model problem set up

\subsection{Confined Vapour Model}

If it is assumed that the water vapour is confined to the inclusion, the maximum pressure will occur when all of the water, in the inclusion, has evaporated. At the time of entrainment there are no vapour molecules present in the slurry. When maximum pressure is achieved the amount of water vapour (in moles, $n$ ) will be equal to the moles of liquid water present at the time of entrainment and this can be calculated

$$
n_{v}=n_{l}
$$




$$
\begin{aligned}
n_{l} & =\frac{m_{l}}{M} \\
& =\frac{4 \pi R_{1}^{3} \phi_{r} \rho_{l}}{3 M},
\end{aligned}
$$

where $R_{1}$ is the inclusion radius, $\phi_{r}$ is the inclusion porosity, $\rho_{l}$ is the density of liquid water, $M$ is the molecular mass of water and $m_{l}$ is the mass of liquid water at the time of entrainment. The maximum pressure can be calculated, using the ideal gas law, as follows:

$$
\begin{aligned}
P & =\frac{n R T}{V}, \\
& =\frac{\rho_{l} R T}{M}, \\
& \approx 600 \mathrm{MPa},
\end{aligned}
$$

where $P$ is the pressure, $R$ is the gas constant, $T$ is the temperature of the inclusion, which is estimated by the magma temperature $\left(T_{m} \approx 1273 \mathrm{~K}\right)$, and $V$ is the volume of the inclusion. The pressure calculated in the confined vapour model is 300 times the tensile strength of the magma ball (2MPa) and this would indicate that the magma would always fragment. However there is evidence of surviving ejecta and therefore the assumption that the vapour is confined cannot be made.

\subsection{Biot Number}

To model Surtseyan ejecta the thermal behaviour of the inclusion needs to be considered. In the transient model it would be convenient if the inclusion could be considered to heat in a uniform manner. The Biot number represents the ratio of the heat transfer resistance inside of, and at the surface of a body. This ratio can be used to determine if the temperature will significantly vary with position inside the body as a temperature gradient is applied to the surface. If a body is calculated to have a small Biot number (less than 1) it can be assumed that inside the body it is heating uniformly. If the Biot number is greater than one we can not assume this.

The Biot number $\left(B_{i}\right)$ is

$$
B_{i}=\frac{h L_{c}}{K_{b}}
$$


where $h$ is the heat transfer coefficient of the surroundings, $L_{c}$ is the characteristic length of the object and $K_{b}$ is the thermal conductivity of the object. To find the value of the Biot number for a Surtseyan ejecta the heat transfer coefficient must be estimated. The heat transfer coefficient is calculated using the formula

$$
h=\frac{Q}{A} \frac{1}{(-\Delta T)}
$$

where $\frac{Q}{A}$, the heat flow per unit area, is calculated using the heat flux conduction equation

$$
\frac{Q}{A}=-K_{2} \frac{d T}{d r}
$$

where $K_{2}$ is the magma's thermal conductivity which is a known constant. A approximate estimate for $\frac{d T}{d r}$ is given by the change in temperature $(\Delta T)$ over the change in radius $(\Delta r)$. The change in radius can be approximatly estimated to be the characteristic length, $L_{2}$, of the magma ball. This is a sufficient approximation for the Biot number but a more accurate approximation will be needed in the thermal equation. Using the estimate for $\frac{d T}{d r}$ the heat flow per unit area is

$$
\frac{Q}{A}=-K_{2} \frac{\Delta T}{L_{2}}
$$

and the heat transfer coefficient, $h$, is

$$
\begin{aligned}
h & =\frac{Q}{A} \frac{1}{(-\Delta T)}, \\
& =\frac{K_{2}}{L_{2}} .
\end{aligned}
$$

The Biot number can be found by assuming that $K_{1} \approx K_{2}$, which is a reasonable assumption as the sediment and the magma are made up of the same type of rock. Then using the definition for characteristic length, $L_{c}=\frac{V_{c}}{A_{c}}=\frac{R_{c}}{3}$, we find that

$$
\begin{aligned}
B_{i} & =\frac{K_{2} L_{1}}{K_{1} L_{2}}, \\
& =\frac{\frac{R_{1}}{3}}{\frac{R_{2}}{3}}, \\
& =\frac{R_{1}}{R_{2}} .
\end{aligned}
$$


As the entrained slurry is enclosed in a ball of magma the Biot number is always less than 1 . Therefore the inclusion can be considered as heating uniformly. Now a thermal equation for the Surtseyan ejecta is needed.

\subsection{The Thermal equation}

To model the thermal system it is assumed that the heating of the entrained slurry is due only to conductive heat transport between the magma and the inclusion. It is also assumed that the radius of the entrained slurry, $R_{1}$, is small compared to the radius of the magma ball, $R_{2}$. As a result the initial temperature of the magma, $T_{m}$, does not vary significantly in the time it takes for the water in the slurry to evaporate. In our model the origin of the radius is considered to be at the centre of the ball, and the time is considered to be zero when the slurry is entrained. Assuming that the amount of heat diffusing out of the magma is equivalent to the amount of heat diffusing into the inclusion a heat balance can be used to estimate the flow of heat into the entrained slurry

$$
\frac{\partial Q}{\partial t}=K_{2} 4 \pi R_{1}^{2} \frac{\partial T_{m}}{\partial r}
$$

The rate of change of the internal energy of the inclusion is assumed to be equivalent the to flow of heat into the slurry

$$
\frac{\partial Q}{\partial t}=\frac{4}{3} \pi R_{1}^{3} \rho c_{p} \frac{\partial T}{\partial t}
$$

where the effective density of the slurry is $\rho=\phi_{r} \rho_{l}+\left(1-\phi_{r}\right) \rho_{m}$. The effective density includes $\rho_{l}$, the density of the liquid water, $\rho_{m}$ is the density of the solid magma and $\phi$ is the porosity of the entrained slurry. To simplify the model we would like to consider the thermal problem separately from the pressure. Heat capacity varies with temperature and pressure. At the boiling point of water the heat capacity increases due to the specific heat of vaporisation of the water. If heat capacity is considered to be the effective heat capacity at constant pressure, $c_{p}$, this allows for the pressure and temperature equations to be considered separately.

Using Equations (2.14) and (2.15) an equation for the temperature at the surface 
of the inclusion, $T$, can be calculated

$$
K_{2} 4 \pi R_{1}{ }^{2} \frac{\partial T_{m}}{\partial r}=\frac{4}{3} \pi R_{1}^{3} \rho c_{p} \frac{\partial T}{\partial t} .
$$

Equation 2.16) simplifies to

$$
\frac{3 K_{2}}{\rho c p R_{1}} \frac{\partial T_{m}}{\partial r}=\frac{\partial T}{\partial t} .
$$

An estimate for $\frac{\partial T_{m}}{\partial r}$ is required to solve the differential equation for $T$. There is a temperature gradient between the magma and the inclusion's varying surface temperature. Over the times taken to heat the inclusion it is reasonable to estimate the length scale as the radius of the inclusion. This gives equation

$$
\frac{3 K_{2}}{\rho c p R_{1}^{2}}\left(T_{m}-T\right)=\frac{\partial T}{\partial t}
$$

which can be solved as the equation is separable

$$
\begin{aligned}
\frac{3 K_{2}}{\rho c p R_{1}^{2}} d t & =\frac{d T}{\left(T_{m}-T\right)}, \\
\int_{0}^{t} \frac{3 K_{2}}{\rho c p R_{1}^{2}} d t & =\int_{T_{0}}^{T} \frac{d T}{\left(T_{m}-T\right)}, \\
\frac{-3 K_{2} t}{\rho c p R_{1}^{2}} & =\ln \left(\frac{T_{m}-T}{T_{m}-T_{0}}\right), \\
T & =T_{m}-\left(T_{m}-T_{0}\right) e^{-\alpha t}
\end{aligned}
$$

where the value $T_{0}$ represents the initial temperature of the slurry when entrained, and

$$
\alpha=\frac{3 K_{2}}{\rho c p R_{1}^{2}}
$$

So in our model the temperature at the surface of the entrained slurry increases and approaches the magma temperature with a time-scale of $\frac{1}{\alpha}$. 


\subsection{Amount of heat required to reach equilibrium}

The amount of heat transferred into the entrained slurry to reach equilibrium, is a value which will be needed to calculate the velocity of the steam generation boundary in Section 2.6. This value will be used to estimate the amount of magma driving the temperature changes in the ball.

The amount of heat required for the inclusion to reach an equilibrium temperature, assuming that the magma remains at a constant temperature, $T_{m}$, is calculated in 3 stages. The first stage is heating the water in the inclusion, from the initial temperature $T_{0}$ to $373 \mathrm{~K}$. As this flashing process occurs quickly it is assumed that the pressure is still equal to 1 bar. The water in the inclusion changing phase from liquid to gas is the second stage. The finial stage is heating the water vapour from $373 \mathrm{~K}$ to $T_{m}$.

The amount of heat needed to heat the liquid water in the inclusion to $373 \mathrm{~K}, Q_{1}$, is

$$
\begin{aligned}
Q_{1} & =\phi_{r} \rho_{l} V c_{p} \Delta T, \\
& =\phi_{r} \rho_{l} \frac{4 \pi}{3} R_{1}^{3} 4187 \times\left(373-T_{0}\right), \\
& =\left(1.8 \times 10^{4}\right)\left(373-T_{0}\right) \phi_{r} \rho_{l} R_{1}^{3}(2 s f) \mathrm{J},
\end{aligned}
$$

where $c_{p}$ is the specific heat of water, $4184 \mathrm{Jkg}^{-1} \mathrm{~K}^{-1}$. The specific heat of water describes the amount of energy needed to heat $1 \mathrm{~kg}$ of water by $1 \mathrm{~K}$. Equation (2.24) also includes the volume of the inclusion, $V$, the porosity of the inclusion, $\phi_{r}$, the density of water, $\rho_{l}$, and the initial temperature of the inclusion, $T_{0}$.

The amount of heat required to vaporise the water is

$$
\begin{aligned}
Q_{2} & =h_{v l} \phi_{r} \rho_{l} V \\
& =\left(9.47 \times 10^{6}\right) \phi_{r} \rho_{l} R_{1}^{3} \mathrm{~J}
\end{aligned}
$$

which includes $h_{v l}$ the latent heat of vaporisation, $2260 \mathrm{~kJ} \mathrm{~kg}^{-1}$. The latent heat of vaporisation of water describes the amount of energy needed to convert $1 \mathrm{~kg}$ of water into steam. 
$Q_{3}$ is the amount of heat needed to change the temperature of the vapour from $373 \mathrm{~K}$ to $T_{m}$. To find out the amount of energy required the specific heat of water vapour is needed. The specific heat of water vapour varies with pressure and temperature. In Table 2.4 there is a range of pressures and temperatures that could occur in the inclusion and their corresponding specific heats. The maximum pressure that can occur in the inclusion is the tensile strength of the magma, which we take here to be $2 \mathrm{MPa}$, because after this pressure is exceeded the ejecta will explode.

\begin{tabular}{|l|l|l|}
\hline \multicolumn{3}{|c|}{ Specific Heat $\left(c_{p}\right)$ at changing Temperatures and Pressures } \\
\hline Temperature $(\mathbf{K})$ & Pressure $(\mathbf{M P a})$ & Specific Heat $\left(\mathbf{J ~ k g}^{-1} \mathbf{K}^{-1}\right)$ \\
\hline 373 & 0.1 & 2042 \\
473 & 0.5 & 2138 \\
573 & 1 & 2147 \\
673 & 1 & 2132 \\
773 & 1 & 2168 \\
873 & 1 & 2224 \\
973 & 1 & 2287 \\
1073 & 1 & 2353 \\
1173 & 1 & 2419 \\
1273 & 2 & 2490 \\
\hline
\end{tabular}

Table 2.1: Values of the specific heat of water vapour at different pressures and temperatures [4].

The specific heat from Table 2.4 and the corresponding temperature can be used to estimate $Q_{3}$. The trapezoidal rule is used to integrate the specific heat with respect to temperature,

$$
\begin{aligned}
\int_{373}^{T_{m}} c p(T) d T & \approx \frac{\left(T_{m}-373\right)}{2 \times 9}(2042+2(2138+2147+2132 \\
& +2168+2224+2287+2353+2419)+2490) \\
& \approx\left(T_{m}-373\right) \times 2237 \mathrm{~J} \mathrm{~kg}^{-1}
\end{aligned}
$$

$Q_{3}$ is then calculated using a similar method to Equation (2.24),

$$
Q_{3}=\phi_{r} \rho_{l} V c_{p} \Delta T
$$




$$
\begin{aligned}
& =\phi_{r} \rho_{l} \frac{4 \pi}{3} R_{1}^{3}\left(T_{m}-373\right) \times 2237 \\
& =\left(9.4 \times 10^{3}\right)\left(T_{m}-373\right) \phi_{r} \rho_{l} R_{1}^{3}(2 s f) \mathrm{J} .
\end{aligned}
$$

The results of $Q_{1}, Q_{2}$ and $Q_{3}$ are combined to find an estimate of the heat transferred into the inclusion for its temperature to reach equilibrium ,

$$
Q=Q_{1}+Q_{2}+Q_{3}=\left(19.37 \times 10^{6}\right) \phi_{r} \rho_{l} R_{1}^{3}(2 s f) \mathrm{J},
$$

it is assumed that $T_{0}=293$ and $T_{m}=1273$. Table 2.4 shows typical values for $Q_{i}$ at a variety of different inclusion sizes assuming that $\phi_{r}=0.4$ and $\rho_{l}=999.97 \mathrm{~kg}$ $\mathrm{m}^{-3}$.

\begin{tabular}{|l|l|l|l|l|}
\hline \multicolumn{5}{|c|}{ Typical values of $Q_{i}$ at different inclusion sizes } \\
\hline$R_{1}(\mathrm{~m})$ & $Q_{1}(\mathrm{~J})$ & $Q_{2}(\mathrm{~J})$ & $Q_{3}(\mathrm{~J})$ & $Q(\mathrm{~J})$ \\
\hline 0.0001 & $5.76 \times 10^{-4}$ & $3.79 \times 10^{-3}$ & $3.38 \times 10^{-3}$ & $7.75 \times 10^{-3}$ \\
0.001 & 0.576 & 3.79 & 3.38 & 7.75 \\
0.01 & 576 & 3790 & 3380 & 7750 \\
0.1 & $576 \times 10^{3}$ & $379 \times 10^{4}$ & $338 \times 10^{4}$ & $775 \times 10^{4}$ \\
1 & $576 \times 10^{6}$ & $379 \times 10^{7}$ & $338 \times 10^{7}$ & $775 \times 10^{7}$ \\
\hline
\end{tabular}

Table 2.2: Values for $Q_{i}$ and the amount of energy needed to reach equilibrium for different inclusion sizes.

The energy needed to change phase and to heat the magma to $1273 \mathrm{~K}$ is of the same order of magnitude. However the energy needed to heat the water to $373 \mathrm{~K}$ is negligible when the total amount of heat needed to reach equilibrium is considered.

\subsection{Estimating the temperature gradient}

In Section 2.6 a better estimate for the temperature gradient is required. The temperature gradient can be estimated by the change in temperature of the inclusion divided by a length-scale, $\Delta r$. In Section 2.2 this length-scale was the radius of the inclusion. To find a better estimate for the temperature gradient a more accurate length-scale is required.

To estimate the new length-scale we need to consider the amount of heat needed 
to vaporise water and how much magma is required to provide this heat. In Section 2.4 the amount of energy needed to vaporise the water in the inclusion is calculated. If we consider $T_{0}=293 \mathrm{~K}$ the energy required is,

$$
\begin{aligned}
Q_{1} & =\phi_{r} \rho_{l} V c_{p} \Delta T \\
& =\left(1.44 \times 10^{6}\right) \phi_{r} \rho_{l} R_{1}^{3}(2 s f) \mathrm{J} \\
Q_{2} & =\left(9.47 \times 10^{6}\right) \phi_{r} \rho_{l} R_{1}^{3} \\
Q_{v} & =Q_{1}+Q_{2} \\
Q_{v} & =\left(10.87 \times 10^{6}\right) \phi_{r} \rho_{l} R_{1}^{3}
\end{aligned}
$$

where $Q_{v}$ is the amount of energy needed to vaporise the water in the inclusion. We have previously assumed that all the energy heating the inclusion must be provided by the magma. Using this assumption and Equation (2.39) the volume of magma required to vaporise the water can be calculated

$$
\begin{aligned}
\left(10.87 \times 10^{6}\right) \phi_{r} \rho_{l} R_{1}{ }^{3} & =V_{m} \phi_{m} \rho_{m} C_{m} \Delta T \\
1.086 \times 10^{10} \frac{\phi_{r}}{\phi_{m}} R_{1}{ }^{3} & =V_{m} \times 2.352 \times 10^{9} \\
V_{m} & =4.62 \frac{\phi_{r}}{\phi_{m}} R_{1}^{3}
\end{aligned}
$$

using the typical values for the density of water $\left(\rho_{l}\right)$ and magma $\left(\rho_{m}\right)$ as well as the heat capacity of magma $\left(C_{m}\right)$ that can be found in Table 2.5 .

\begin{tabular}{|l|l|l|}
\hline \multicolumn{3}{|c|}{ Typical Values } \\
\hline Parameter & Value & Units \\
\hline$\rho_{l}$ & 999.97 & $\mathrm{~kg} \cdot \mathrm{m}^{-3}$ \\
$\rho_{m}$ & 2800 & $\mathrm{~kg} \cdot \mathrm{m}^{-3}$ \\
$C_{m}$ & $0.84 \times 10^{3}$ & $\mathrm{~J} \cdot \mathrm{kg}^{-1} \cdot \mathrm{K}^{-1}$ \\
\hline
\end{tabular}

Table 2.3: Typical parameter values.

This magma is in a spherical shell surrounding the inclusion. The volume of this shell is

$$
V_{m}=\frac{4 \pi}{3}\left(R_{\text {used }}+R_{1}\right)^{3}-\frac{4 \pi}{3} R_{1}^{3},
$$

where $R_{\text {used }}$ is the radius of magma used to heat the inclusion. $R_{\text {used }}$ is an 
estimate for the length-scale at short times. By equating Equations 2.42) and (2.43) $R_{\text {used }}$ is calculated

$$
\begin{aligned}
& V=4.62 \frac{\phi_{r}}{\phi_{m}} R_{1}^{3}, \\
\Rightarrow & \frac{4 \pi}{3}\left(R_{\text {used }}+R_{1}\right)^{3}-\frac{4 \pi}{3} R_{1}^{3}=4.62 \frac{\phi_{r}}{\phi_{m}} R_{1}^{3}, \\
\Rightarrow & \left(R_{\text {used }}+R_{1}\right)^{3}-R_{1}^{3}=1.13 \frac{\phi_{r}}{\phi_{m}} \times R_{1}^{3}, \\
\Rightarrow & \left(R_{\text {used }}+R_{1}\right)^{3}=\left(1.13 \frac{\phi_{r}}{\phi_{m}}+1\right) \times R_{1}^{3}, \\
\Rightarrow & \left(R_{\text {used }}+R_{1}\right)=\left(1.13 \frac{\phi_{r}}{\phi_{m}}+1\right)^{\frac{1}{3}} \times R_{1}, \\
\Rightarrow & R_{\text {used }}=\left(\left(1.13 \frac{\phi_{r}}{\phi_{m}}+1\right)^{\frac{1}{3}}-1\right) \times R_{1} .
\end{aligned}
$$

The entrained slurry is comprised of cold bubbly (vesicular) magma that was soaked with water before being washed back into the volcano, it can therefore be assumed that the porosity of the inclusion is equal to that of the magma. When this is assumed $R_{\text {used }}=0.28 R_{1}$ and the temperature gradient can be estimated to be

$$
\begin{aligned}
\frac{\partial T}{\partial r} & \approx \frac{T_{m}-T}{0.28 R_{1}} \\
& \approx \frac{\left(T_{m}-T_{0}\right) e^{-\alpha t}}{0.28 R_{1}}
\end{aligned}
$$

which will be used in the boundary condition for the pressure model.

\subsection{Pressure Equations}

The pressure variations in this system are driven by vapour generation and the diffusion of vapour through the pores of the surrounding magma. In geothermal literature there are models for water vapour flowing through a porous medium ([22] [11]). We have adapted a geothermal approach to suit the Surtseyan ejecta problem.

In Pruess's geothermal injection model [22], to construct the pressure equation, a 
conservation of vapour mass equation is considered

$$
\frac{\partial\left(\phi_{m} \rho_{v}\right)}{\partial t}=-\nabla \cdot \mathbf{F}_{v}
$$

The mass flux $\left(\mathbf{F}_{v}\right)$ is calculated using Darcy's law

$$
\mathbf{F}_{v}=-k \frac{\rho_{v}}{\mu_{v}} \nabla p
$$

Darcy's law describes fluid flow in a porous media. We are assuming that only vapour flows through the pores of the magma. In the Surtseyan ejecta problem this describes the water vapour escaping through the vesicular magma. By combining Equation 2.53) and 2.52) we obtain

$$
\frac{\partial\left(\phi_{m} \rho_{v}\right)}{\partial t}=\frac{k}{\mu_{v}} \nabla \cdot\left(\rho_{v} \nabla p\right) .
$$

The density of the gas depends on pressure through the ideal gas law. In the Surtseyan ejecta problem we will ignore the temperature variation and assume that the vapour when flowing through the magma has already reached the temperature of the magma $T_{m}$. To use this technique we are assuming that the water vapour acts like an ideal gas. Using the ideal gas law we obtain:

$$
\begin{aligned}
& \frac{\phi_{m} M}{R T_{m}} \frac{\partial p}{\partial t}=\frac{k}{\mu_{v}} \nabla \cdot\left(\frac{M p}{R T_{m}} \nabla p\right), \\
\Rightarrow & \phi_{m} \frac{\partial p}{\partial t}=\frac{k}{\mu_{v}} \nabla \cdot(p \nabla p), \\
\Rightarrow & \frac{\partial p}{\partial t}=\frac{k}{\mu_{v} \phi_{m}} \nabla\left(\frac{1}{2} \nabla p^{2}\right) .
\end{aligned}
$$

Equation 2.57) can be expressed in spherical polar coordinates as

$$
\begin{aligned}
\frac{\partial p}{\partial t} & =\frac{1}{r^{2}} \frac{\partial}{\partial r}\left[D r^{2} \frac{\partial p^{2}}{\partial r}\right] \\
D & =\frac{k}{2 \phi_{m} \mu_{v}} .
\end{aligned}
$$

This is a non-linear diffusion equation for the vapour pressure. The initial 
condition is that the partial pressure of water in the vesicular magma is negligible at the time of entrainment $p(r, 0)=0$. The total pressure at the surface of the ejecta is assumed to be one atmosphere. The partial pressure of water vapour at the surface can range up to 1 atmosphere, which is much smaller that the critical fragmentation pressure $\left(p_{c}\right)$. Therefore the boundary condition at the surface of the magma ball is assumed to also correspond to negligible partial pressure of water vapour

$$
p=0, \quad r=R_{2}
$$

For the second boundary condition the steam generation boundary in the entrained slurry is considered. The flow of heat entering the inclusion vaporises the water resulting in the pressure build up. In the model we are considering the steam generation boundary to be propagating into the inclusion at a rate governed by the temperature gradient.

The following assumptions are made:

- The slurry starts to boil at the surface but the temperature of the slurry heats in a uniform manner (Biot Number from Section 2.2.

- The boiling front moves towards the centre of the inclusion with time

- The large temperature gradient causing the boiling of the vapour has a much larger effect than the steam removal and energy balance processes.

Using the assumption that the heat is driving the change in enthalpy in the problem it can be assumed that

$$
(\text { Heat In }- \text { Heat out })=\Delta \text { enthalpy }
$$

The amount of heat that is transferred into the inclusion in the time $\Delta t$ is

$$
\left.4 \pi s^{2} K \frac{\partial T}{\partial r}\right|_{s} \Delta t
$$

and the heat escaping the inclusion in this time is assumed to be negligible. In Equation 2.62 it is assumed that $\frac{\partial T}{\partial r}$ is a know value (derived in Section 2.5 . 


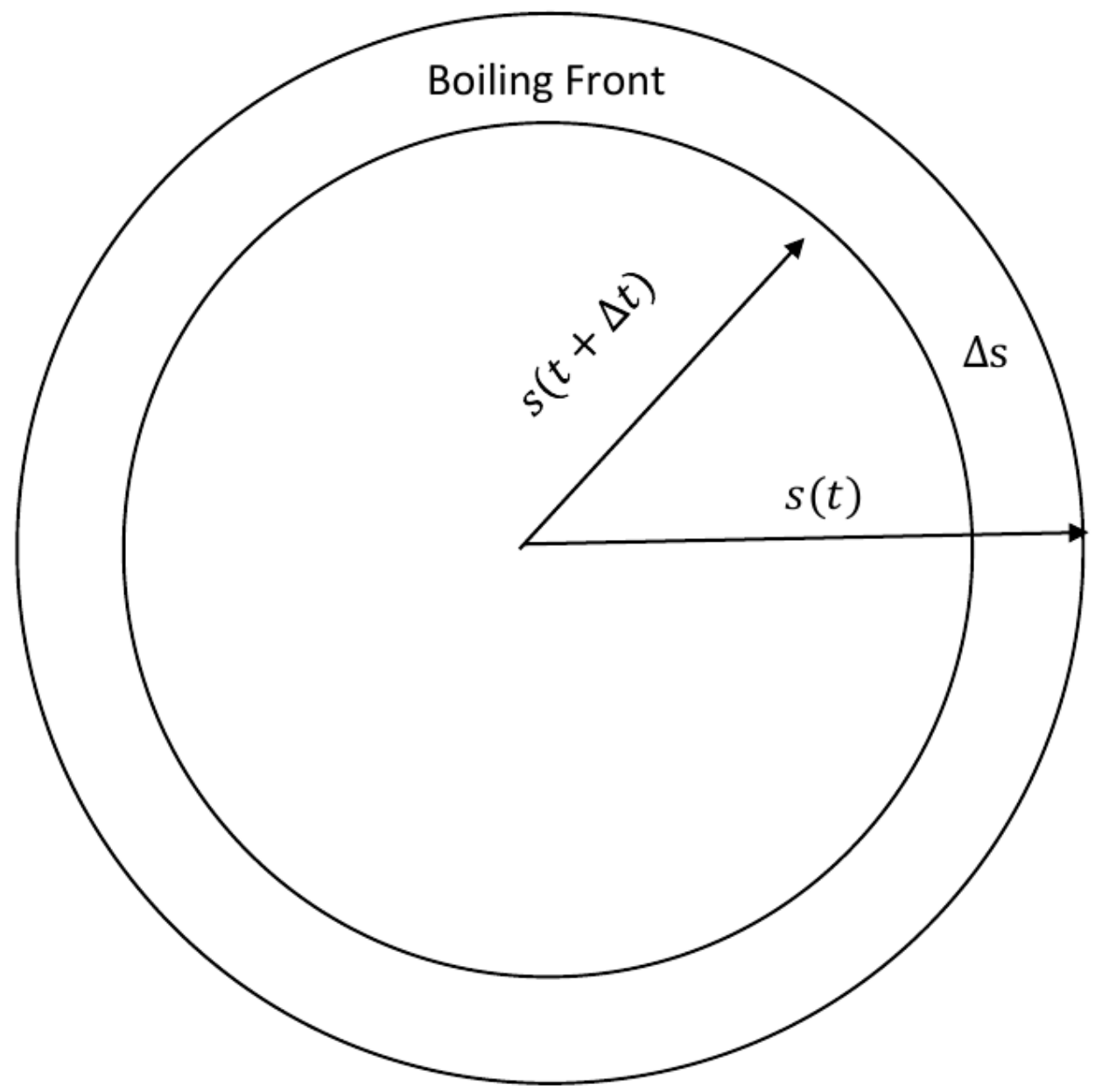

Figure 2.2: Diagram depicting the movement of the steam generation boundary

The change of enthalpy is

$$
\frac{4 \pi}{3}\left[s^{3}(t)-s^{3}(t+\Delta t)\right] \rho_{l} \phi h_{v l}
$$

The enthalpy change is derived by considering the amount of energy needed for the water swept by the steam generation boundary in time $\Delta t$ to vaporise. This can be seen in Figure 2.2. The change in enthalpy and the heat transfer can be substituted into Equation (2.61) to obtain

$$
\left.4 \pi s^{2} K \frac{\partial T}{\partial r}\right|_{s} \Delta t-0=\left(\frac{4 \pi}{3}\left[s^{3}(t)-s^{3}(t+\Delta t)\right] \rho_{l} \phi h_{v l}\right)
$$




$$
\left.\Rightarrow K \frac{\partial T}{\partial r}\right|_{s}=\frac{\left(\frac{1}{3}\left[s^{3}(t)-s^{3}(t+\Delta t)\right] \rho_{l} \phi h_{v l}\right)}{s^{2} \Delta t} .
$$

Using the formal definition of a derivative

$$
f^{\prime}(x)=\lim _{h \rightarrow 0} \frac{f(x+h)-f(x)}{h},
$$

and by the taking the limit as $\Delta t$ tends to zero, Equation (2.64) can be written in terms of the velocity of the steam generation boundary

$$
\begin{aligned}
& \left.K \frac{\partial T}{\partial r}\right|_{s}=\frac{\left(\frac{1}{3}\left[-3 s^{2} \dot{s}\right] \rho_{l} \phi h_{v l}\right)}{s^{2}} \\
\Rightarrow & \left.K \frac{\partial T}{\partial r}\right|_{s}=-\left(\dot{s} \rho_{l} \phi h_{v l}\right), \\
\Rightarrow & \dot{s}=-\left.\frac{K}{\rho_{l} \phi h_{v l}} \frac{\partial T}{\partial r}\right|_{s}
\end{aligned}
$$

An equation for the position of the steam generation boundary is obtained using Equation 2.69) and the initial condition $s=R_{1}$ at $t=0$. This is achieved by integrating 2.69) with respect to time, while assuming that the temperature gradient is $\left.\frac{\partial T}{\partial r}\right|_{S} \approx \frac{\left(T_{m}-T_{0}\right) e^{-\alpha t}}{0.28 R_{1}}$ which is derived in Section 2.5

$$
\begin{aligned}
& d s=-\left.\frac{K}{\rho_{l} \phi h_{v l}} \frac{\partial T}{\partial r}\right|_{s} d t \\
\Rightarrow & d s=-\frac{K}{\rho_{l} \phi h_{v l}} \frac{\left(T_{m}-T_{0}\right) e^{-\alpha t}}{0.28 R_{1}} d t \\
\Rightarrow & -\int_{s}^{R 1} d s=\int_{0}^{t}-\frac{K}{\rho_{l} \phi h_{v l}} \frac{\left(T_{m}-T_{0}\right) e^{-\alpha t}}{0.28 R_{1}} d t \\
\Rightarrow \quad & s=B\left(e^{-\alpha t}-1\right)+R_{1} \\
\Rightarrow & B=\frac{\left(T_{m}-T_{0}\right) \rho c_{p} R_{1}}{0.28 \times 3 \rho_{l} \phi h_{v l}}
\end{aligned}
$$

The critical time $t_{c}$, the time at which the water is completely depleted, can be calculated by setting Equation (2.73) to zero resulting in

$$
t_{c}=-\frac{1}{\alpha} \ln \left(1-\frac{R_{1}}{B}\right) .
$$

The velocity of the steam generation boundary, $\dot{s}$, can be used to determine the 
pressure boundary condition at the surface $s$. If we consider the rate of change of water volume swept by the boiling front

$$
\frac{d V_{w a t e r}}{d t}=4 \pi s^{2}(|\dot{s}|)
$$

this can be used to obtain the rate of change of the mass of water swept by the steam generation boundary. The rate of change of the mass of water swept by the boiling front must be equal to the rate of change of vapour produced by the steam generation boundary, due to conservation of mass in the system. The rate of change of vapour produced by the steam generation boundary is

$$
\frac{d m_{\text {vapour }}}{d t}=4 \pi s^{2}|\dot{s}| \phi \rho_{l}
$$

and this can be used to find the rate of change of vapour volume produced by the steam generation boundary

$$
\frac{d V_{\text {vapour }}}{d t}=4 \pi s^{2}|\dot{s}| \phi \frac{\rho_{l}}{\rho_{v}}
$$

Using Equation (2.69) and Darcy's law, Equation 2.53), together with the ideal gas law (to replace the density of the water vapour) the flux boundary condition at the surface of the entrained slurry can be written as

$$
\begin{aligned}
& \frac{d V_{\text {vapour }}}{d t}=\left.\frac{k A}{\mu_{v}} \nabla p\right|_{r=s}, \\
\Rightarrow & 4 \pi s^{2}|\dot{s}| \phi \rho_{l} \frac{R T_{m}}{M p}=\left.\frac{k 4 \pi s^{2}}{\mu_{v}} \nabla p\right|_{r=s}, \\
\Rightarrow & \left.p \nabla p\right|_{r=s}=\frac{\mu_{v} \phi \rho_{l}|\dot{s}| R T_{m}}{M k}, \\
\Rightarrow & \left.\nabla p^{2}\right|_{r=s}=\frac{2 \mu_{v} \phi \rho_{l}|\dot{s}| R T_{m}}{M k} .
\end{aligned}
$$

This gives the mass flux at the steam generation boundary

$$
\left.\left(\frac{k}{2 \phi_{m} \mu_{v}}\right) \nabla p^{2}\right|_{r=s}=\frac{\phi \rho_{l}|\dot{s}| R T_{m}}{M \phi_{m}} .
$$

When the steam generation boundary is between the origin and $R_{1}$ Equation 
(2.83) is the boundary condition. However after the critical time, when there is no more liquid vaporising in the slurry, the boundary condition at the origin is

$$
\left(\frac{k}{2 \phi_{m} \mu_{v}}\right) \nabla p^{2}=0
$$

To summarise the dimensional problem to solve is,

$$
\begin{aligned}
\frac{\partial p}{\partial t} & =\frac{1}{r^{2}} \frac{\partial}{\partial r}\left[D r^{2} \frac{\partial p^{2}}{\partial r}\right] \\
p & =0, r=R_{2} \\
D \frac{\partial p^{2}}{\partial r} & =\left\{\begin{array}{ll}
\frac{\phi \rho_{l}|s| R T_{m}}{M \phi_{m}} & : t<t_{c} \\
0 & : t \geqslant t_{c}
\end{array}, r=s(t)\right. \\
\dot{s} & =-\frac{K\left(T_{m}-T_{0}\right) e^{-\alpha t}}{\rho_{l} \phi h_{v l} \times 0.28 R_{1}} \\
\alpha & =\frac{3 K_{2}}{\rho c p R_{1}^{2}} \\
D & =\frac{k}{2 \phi_{m} \mu_{v}} \\
t_{c} & =-\frac{1}{\alpha} \ln \left(1-\frac{R_{1}}{B}\right) \\
B & =\frac{\left(T_{m}-T_{0}\right) \rho c_{p} R_{1}}{0.28 \times 3 \rho_{l} \phi h_{v l}} .
\end{aligned}
$$

\subsection{Non-Dimensionalisation}

Non-dimensionalisation is used to simplify systems that are too complicated to analyse rigorously. During this process all of the terms are scaled to produce dimensionless variables. By doing this it is possible to compare different components by setting the biggest terms to order one, so that the smaller terms are apparent, this is to ensure that the parameters are combined into appropriate groupings. The purpose in this case is to create a simple model appropriate for numerical simulations and to find the combination of parameters that affect rupture. The challenge with non-dimensionalisation is if it is scaled incorrectly the area of interest can get lost as the relevant information occurs in a really small time span. 
The dimensional problem is non-dimensionalised using the scaling

$$
\tilde{p}=\frac{p}{p_{c}}, \tilde{t}=\frac{t}{t_{c}}, \quad \tilde{r}=\frac{r}{R_{2}}
$$

where $p_{c} \approx 2 \times 10^{6} \mathrm{~Pa}$ is the tensile strength of the magma. When the pressure reaches this value it is expected that the Surtseyan ejecta will rupture. In the rescaling, rupture will occur at $\tilde{p}=1$. A rescaling for the diffusivity is also needed but this will be derived when we apply the rescaling to the model.

The resulting non-dimensionalised pressure diffusion equation is

$$
\frac{\partial \tilde{p}}{\partial \tilde{t}}=\frac{1}{\tilde{r}^{2}} \frac{\partial}{\partial \tilde{r}}\left[\frac{t_{c} p_{c} D}{R_{2}{ }^{2}} \tilde{r}^{2} \frac{\partial \tilde{p}^{2}}{\partial \tilde{r}}\right]
$$

From Equation 2.94) the non-dimensionalised diffusivity can be set to

$$
\tilde{D}=\frac{t_{c} p_{c} D}{R_{2}^{2}}
$$

When the rescaling is applied to the dimensional model, summarized in Equations 2.85) to 2.92, we obtain the following non-dimensional model:

$$
\begin{aligned}
\frac{\partial \tilde{p}}{\partial \tilde{t}} & =\frac{1}{\tilde{r}^{2}} \frac{\partial}{\partial \tilde{r}}\left[\tilde{D} \tilde{r}^{2} \frac{\partial \tilde{p}^{2}}{\partial \tilde{r}}\right] \\
\tilde{p}(\tilde{r}, 0) & =0, \tilde{p}(1, \tilde{t})=0 \\
\tilde{D} \frac{\partial \tilde{p}^{2}}{\partial \tilde{r}} & =\left\{\begin{array}{cc}
C \dot{s} \quad: \tilde{t}<1 \\
0 \quad: \tilde{t} \geqslant 1
\end{array}, \tilde{r}=\frac{s(t)}{R_{2}}\right. \\
\dot{s} & =-\frac{K\left(T_{m}-T_{0}\right) e^{-\alpha t_{c} \tilde{t}}}{\rho_{l} \phi h_{v l} \times 0.28 R_{1}} \\
C & =\frac{t_{c} R T_{m} \phi \rho_{l}}{R_{2} p_{c} M \phi_{m}} .
\end{aligned}
$$




\section{Chapter 3}

\section{Numerical Solutions}

We numerically solve our model for Surtseyan ejecta to determine the behaviour of the pressures in the inclusion. As the model is based on a model for geothermal injection [22], which can be solved analytically . We need to consider whether the Surtseyan ejecta model can be solved in the same manner. In the Surtseyan ejecta model an average pressure cannot be assumed because of the wide range of pressures in the system. This differential equation without this assumption cannot be solved analytically and numerical techniques must be used. MATLAB is used to solve Equations (3.1) to (3.5) numerically:

$$
\begin{aligned}
\frac{\partial \tilde{p}}{\partial \tilde{t}} & =\frac{1}{\tilde{r}^{2}} \frac{\partial}{\partial \tilde{r}}\left[\tilde{D} \tilde{r}^{2} \frac{\partial \tilde{p}^{2}}{\partial \tilde{r}}\right], \\
\tilde{p}(\tilde{r}, 0) & =0, \tilde{p}\left(R_{2}, \tilde{t}\right)=0, \\
\tilde{D} \frac{\partial \tilde{p}^{2}}{\partial \tilde{r}} & =\left\{\begin{array}{cc}
C \dot{s} & : \tilde{t}<1 \\
0 & : \tilde{t} \geqslant 1
\end{array}, \tilde{r}=\frac{s(t)}{R_{2}},\right. \\
\dot{s} & =-\frac{K\left(T_{m}-T_{0}\right) e^{-\alpha t_{c} \tilde{t}}}{\rho_{l} \phi h_{v l} \times 0.28 R_{1}}, \\
C & =\frac{t_{c} R T_{m} \phi \rho_{l}}{R_{2} p_{c} M \phi_{m}} .
\end{aligned}
$$

PDEPE, a standard MATLAB function, is used to solve partial differential equations of the form $c\left(x, t, u, \frac{\partial u}{\partial x}\right) \frac{\partial u}{\partial t}=x^{-m} \frac{\partial}{\partial x}\left(x^{m} f\left(x, t, u, \frac{\partial u}{\partial x}\right)+s\left(x, t, u, \frac{\partial u}{\partial x}\right)\right)$ where the value $m$ is set to 0,1 or 2 depending on the whether the modelled system has slab, cylindrical or spherical symmetries. The problem must also be 
defined over a fixed finite interval for PDEPE to be used. In the programming, $x$ is considered to be the non-dimensionalised radius and $u$ is the non-dimensionalised pressure. The Surtseyan ejecta model, derived in Chapter 2, is already in the appropriate form so $c, f$ and $s$ can be immediately defined as follows:

$$
\begin{aligned}
c & =1, \\
f & =2 u \tilde{D} \frac{\partial u}{\partial x} \\
s & =0 .
\end{aligned}
$$

As PDEPE requires a defined interval, singularities need to be considered. At $\tilde{r}=0$ there is a singularity, dividing by zero, so this point cannot be contained within the interval. Therefore we define the interval to be from a fixed small number (close to 0 ) to 1 , which is at the surface of the magma ball.

The last requirement is for the steam generation boundary to be fixed, as the program used needs fixed boundary conditions. The non-dimensional model has a travelling inner boundary condition. Since $s \leqslant R_{1}<<R_{2}$, this moving boundary can be replaced with a point source of vapour at the origin. However the flux condition is applied at the surface of the inclusion $\tilde{r}=\varepsilon=\frac{R_{1}}{R_{2}}$ which is much smaller than 1 . This results in a fixed boundary condition near the origin but it avoids problems that would arise from the singularity at the origin.

As $\alpha t_{c}$ is very small we can assume that $e^{-\alpha t_{c} \tilde{t}} \approx 1$ when $\tilde{t}<1$. This simplifies the velocity of the steam generation boundary to

$$
\dot{s} \approx-\frac{K\left(T_{m}-T_{0}\right)}{\rho_{l} \phi h_{v l} \times 0.28 R_{1}}
$$

and the critical time to

$$
t_{c}=\frac{0.28 \times \phi R_{1}^{2} \rho_{l} h_{v l}}{K\left(T_{m}-T_{0}\right)}
$$

which is a constant. 
Then the flux boundary condition becomes:

$$
\begin{aligned}
\tilde{D} \frac{\partial \tilde{p}^{2}}{\partial \tilde{r}} & =\left\{\begin{array}{cc}
-E & : \tilde{t}<1 \\
0 & : \tilde{t} \geqslant 1
\end{array}, \tilde{r}=\varepsilon\right. \\
\tilde{D} & =\frac{0.28 \times \phi R_{1}^{2} \rho_{l} h_{v l} p_{c} k}{2 \phi_{m} \mu_{v} K R_{2}^{2}\left(T_{m}-T_{0}\right)}, \\
E & =\frac{R_{1} R T_{m} \phi \rho_{l}}{R_{2} M \phi_{m} p_{c}}
\end{aligned}
$$

The parameter $E$ was found using the distance that the steam generation boundary covers to reach the origin and the required time, $t_{c}$, as well as the parameter $C$. The key parameters controlling the solutions behaviour are the diffusivity, $\tilde{D}$, and the flux at the point source $E$.

The behaviour at the origin in this system is difficult to deduce. As $R_{1}$ increases the flux $E$ at the origin and the diffusivity, $\tilde{D}$ both increase making the behaviour at the origin difficult to predict. The program we used to solve the model numerically is found in Appendix $B$ and the typical values for the parameters used are listed in Table 3.2 . 


\begin{tabular}{|l|l|l|}
\hline \multicolumn{3}{|c|}{ Typical Values } \\
\hline Parameter & Value & Units \\
\hline$p_{c}$ & $2 \times 10^{6}$ & $\mathrm{~Pa}$ \\
$h_{v l}$ & $2.26 \times 10^{6}$ & $\mathrm{~J} \mathrm{~kg}^{-1}$ \\
$\rho_{l}$ & 999.97 & $\mathrm{~kg} \mathrm{~m}^{-3}$ \\
$k$ & $10^{-14}$ & $\mathrm{~m}^{2}$ \\
$K$ & 1.2 & $\mathrm{~W}^{-1} \mathrm{~K}^{-1}$ \\
$\mu_{v}$ & $3 \times 10^{-5}$ & $\mathrm{~Pa} \mathrm{~s}^{-1}$ \\
$M$ & $18 \times 10^{-3}$ & $\mathrm{~kg} \mathrm{~mol}^{-1}$ \\
$R$ & 8.314 & $\mathrm{~J} \mathrm{~K} \mathrm{~mol}^{-1}$ \\
$T_{m}$ & 1273 & $\mathrm{~K}$ \\
$T_{0}$ & 293 & $\mathrm{~K}$ \\
$\phi$ & 0.4 & - \\
$\phi_{m}$ & 0.4 & - \\
$R_{1}$ & 0.001 & $\mathrm{~m}$ \\
$R_{2}$ & 0.1 & $\mathrm{~m}$ \\
\hline
\end{tabular}

Table 3.2: Table containing typical parameter values of a Surtseyan ejecta

\subsection{Results}

The numerical solutions reveal that over a wide range of parameters the pressure at the surface of the entrained slurry increases sharply from zero and then quickly equilibrates. This can be seen clearly in Figure 3.1. This rapid stabilisation means that the maximum pressure can be calculated using the steady state solution for pressure with vapour being produced continuously at the point $\tilde{r}=\varepsilon$. This steady state is not typically reached before the critical time, see Figure 3.1. but if the vapour continued to be produced a steady state would eventually be reached. The pressure at the point $\tilde{r}=\varepsilon$ is equal to the steady state pressure as the model reaches the stable value rapidly.

The steady state solution is

$$
\frac{1}{\tilde{r}^{2}} \frac{\partial}{\partial \tilde{r}}\left[\tilde{D} \tilde{r}^{2} \frac{\partial \tilde{p}^{2}}{\partial \tilde{r}}\right]=0 .
$$




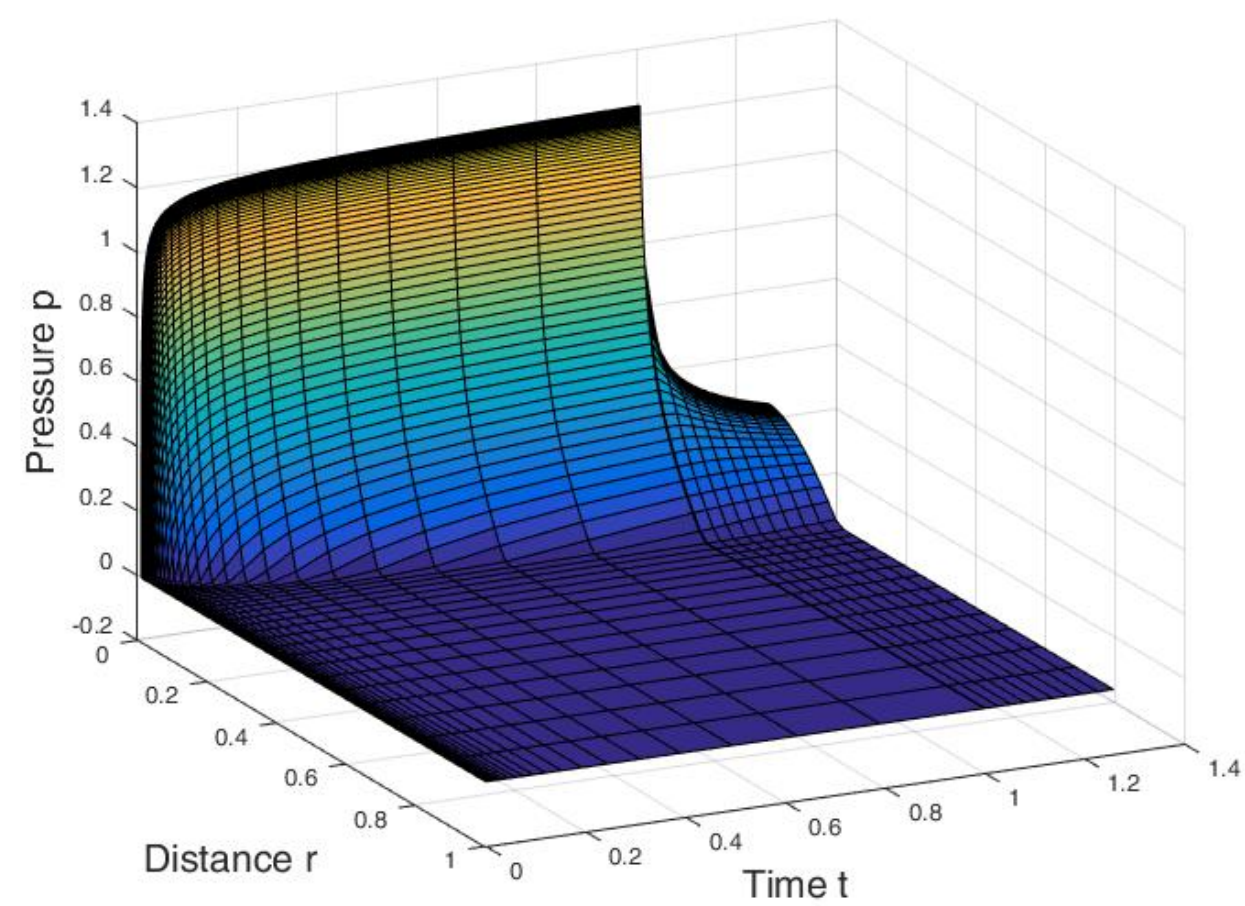

Figure 3.1: Numerical solutions to the non dimensional pressure equations with the parameter as listed in Table 3.2

Equation (3.11) can be solved by integrating twice to for an equation for the steady state pressure

$$
\begin{gathered}
\tilde{r}^{2} \frac{\partial \tilde{p}^{2}}{\partial \tilde{r}}=c_{1}, \\
\Rightarrow \quad \tilde{p}^{2}=-\frac{c_{1}}{\tilde{r}}+c_{2},
\end{gathered}
$$

where $c_{1}$ and $c_{2}$ are the constants of integration. The constants of integration are determined by considering the boundary conditions at $\tilde{r}=\varepsilon$ and $\tilde{r}=1$. By setting $\tilde{r}=1$ it is found that $c_{1}=c_{2}$ and then by considering the flux at $\tilde{r}=\varepsilon$,

$$
\begin{aligned}
& \tilde{D} \frac{\partial \tilde{p}^{2}}{\partial \tilde{r}}=-E, \\
\Rightarrow & \frac{\partial \tilde{p}^{2}}{\partial \tilde{r}}=\frac{-E}{\tilde{D}}, \\
\Rightarrow & \tilde{r}^{2} \frac{\partial \tilde{p}^{2}}{\partial \tilde{r}}=\frac{-E \varepsilon^{2}}{\tilde{D}}=c_{1},
\end{aligned}
$$


the value of $c_{1}$ is determined. The constants are substituted into Equation (3.13) this gives the steady solution

$$
\tilde{p}^{2}=\frac{E \varepsilon^{2}}{\tilde{D}}\left(\frac{1}{\tilde{r}}-1\right)
$$

If the maximum pressure inside a Surtseyan ejecta exceeds the critical pressure the ejecta will rupture. To determine a criterion for rupture a formula for the maximum pressure is required. The maximum pressure occurs at surface of the inclusion, $\tilde{r}=\varepsilon$, at this point the maximum pressure is rapidly approached and can be determined using the steady state equation at $\tilde{r}=\varepsilon$

$$
\begin{aligned}
\tilde{p}(\varepsilon)^{2} & =F(1-\varepsilon), \\
F & =\frac{E \varepsilon}{\tilde{D}} .
\end{aligned}
$$

When the maximum pressure in equation 3.18 exceeds 1 the Surtseyan ejecta is expected to rupture.

The criterion for rupture can be determined from Equation (3.18). If it is assumed that the radius of the inclusion is much smaller than that of the magma ball, then $(1-\varepsilon) \approx 1$, the approximate maximum pressure is $\tilde{p}(\varepsilon)=\sqrt{F}$. Therefore the criterion for rupture is that

$$
\sqrt{\frac{2 R T_{m} \mu_{v} K\left(T_{m}-T_{0}\right)}{M p_{c}^{2} \times 0.28 h_{v l} k}}>1 .
$$

If it cannot be assumed that $(1-\varepsilon) \approx 1$ then the approximate maximum pressure equation is

$$
\tilde{p}(\varepsilon)=\sqrt{F(1-\varepsilon)}
$$

and the criterion for rupture are

$$
\sqrt{\frac{2 R T_{m} \mu_{v} K\left(T_{m}-T_{0}\right)}{M p_{c}^{2} \times 0.28 h_{v l} k}\left(\frac{R_{2}-R_{1}}{R_{2}}\right)}>1 .
$$

The criterion for rupture and the sensitivity analysis (Figure 3.3) allow us to determine which of the parameters affect the maximum pressure. It is interesting to note that when the radius of the inclusion is much smaller than the magma ball 
that the maximum pressure is independent of both $R_{1}$ and $R_{2}$. An example of this can be seen when comparing Figures 3.1 and 3.2. These figures show the pressure distribution in two ejecta that have identical properties apart from a change in $R_{1}$, the maximum pressure in the two figures is very similar, however in 3.2 the steady state is almost reached before the water is depleted.

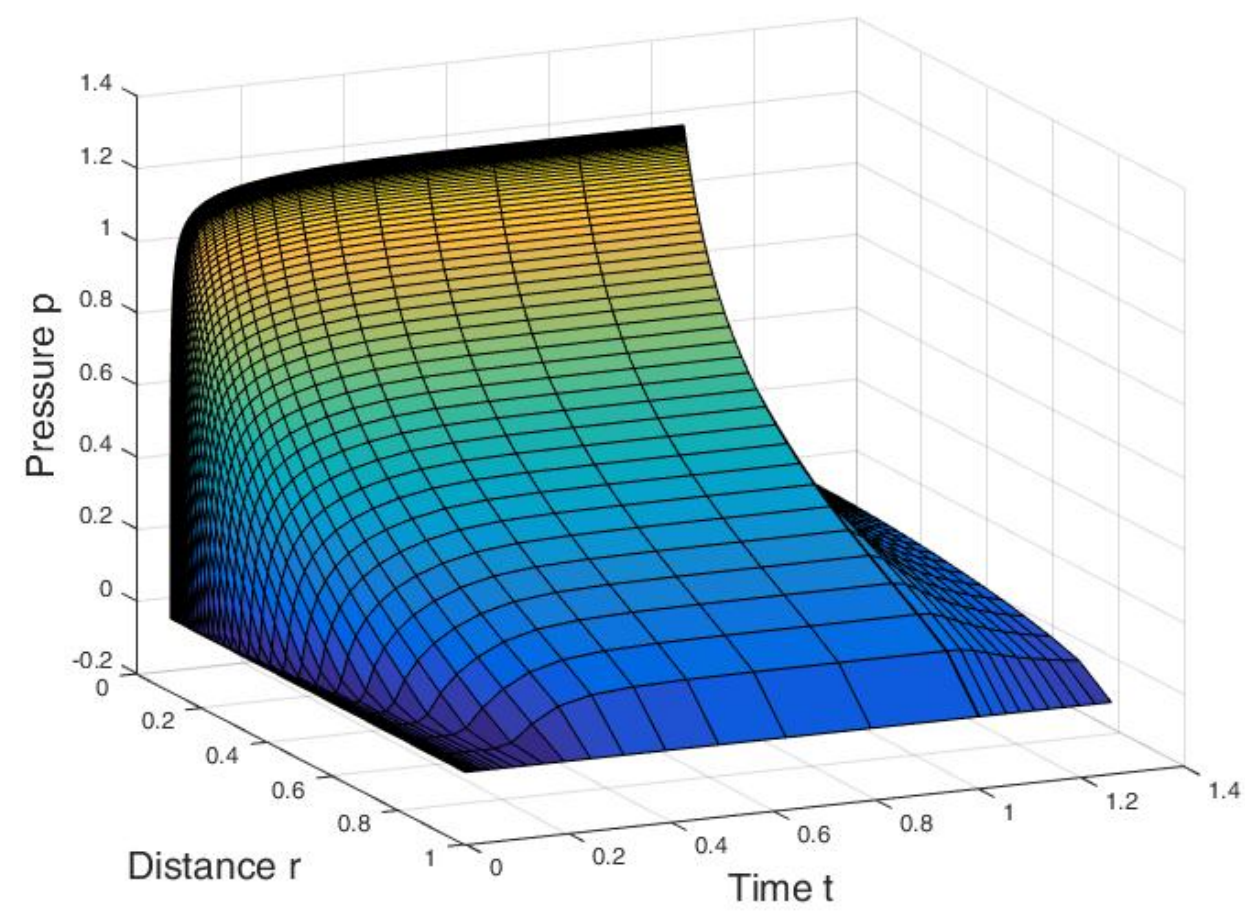

Figure 3.2: Numerical solutions to the non dimensional pressure equations with the parameter as listed in Table 3.2 apart from the radius of the entrained slurry which is $1 \mathrm{~cm}$

The lack of dependence on the inclusion radius, when it is much smaller than the magma, can also be seen in the sensitivity analysis (Figure 3.3). However the sensitivity analysis shows that if the slurry entrainment is a larger fraction of the magma size the pressures will start to decease, this is also apparent from Equation (3.22).

The rupture criterion also has no dependence on either the porosity of the magma or entrained slurry. This is also observed in the sensitivity analysis that shows very small, practically negligible, changes in pressure when the porosity of either 
the magma or the slurry is varied. The porosity of the magma having little affect could be explained by the observed steady state pressure solution, which is independent of the diffusivity, as well as the velocity of the steam generation boundary that does not depend on the magma porosity. The velocity of the steam generation boundary depends on the temperature gradient driving the steam from the magma and the porosity of the inclusion which is the source of the liquid being vaporised. As a result the critical time depends on porosity of the inclusion and this is included in the non dimensionalised diffusivity. The porosity of the inclusion is also present in the parameter $E$ from the rate of change of vapour volume. The porosity of the inclusion appears linear in both $E$ and $\tilde{D}$ and therefore is not present in the criterion for rupture, which depends on the ratio of $E$ and $\tilde{D}$.
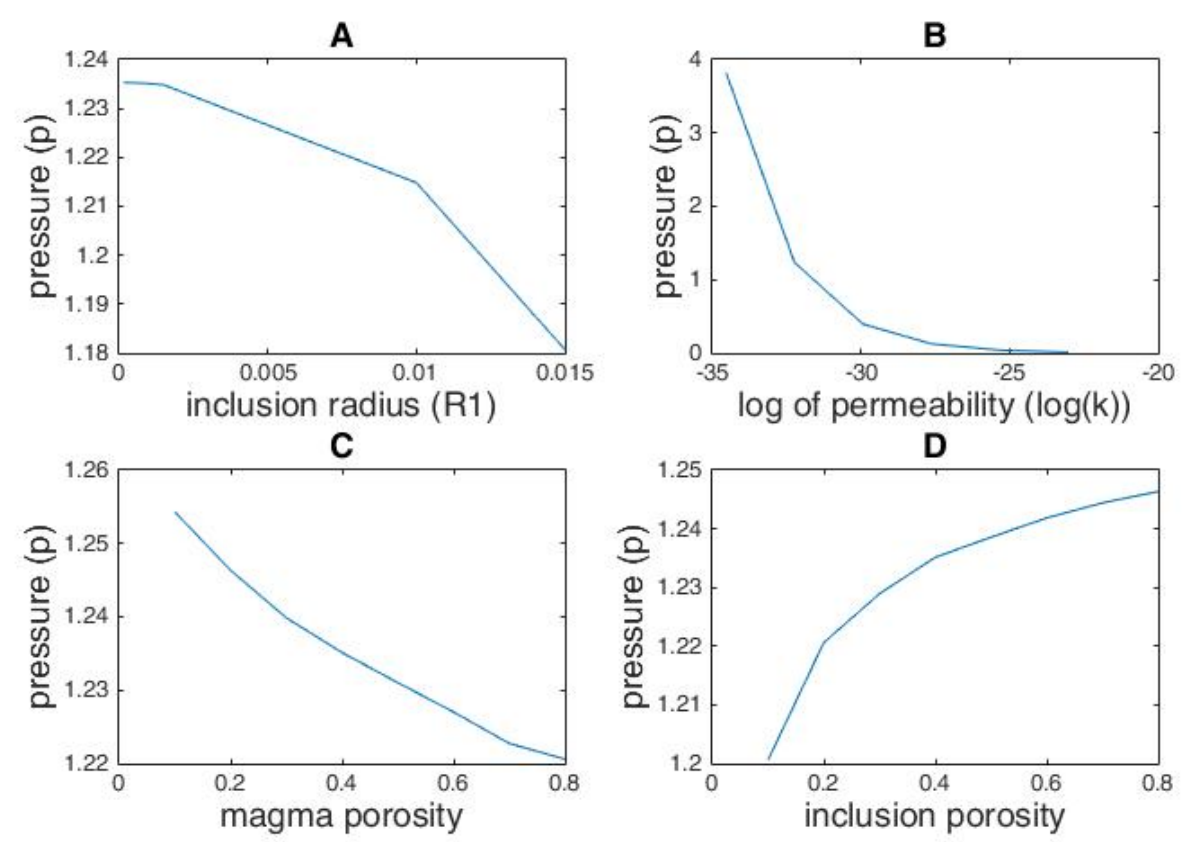

Figure 3.3: Sensitivity analysis for the changes in maximum pressure at the edge of the inclusion when the radius of the entrained slurry (Graph A), permeability of the magma (Graph B) and the porosities of the inclusion (Graph D) and magma (Graph C) are varied

The sensitivity analysis (Figure 3.3) shows how the maximum pressure, at the surface of the inclusion, in the Surtseyan ejecta system is affected by changing one of the parameters. We have studied how varying the inclusion size, the permeability of the magma and the porosity of both the magma and the inclusion 
affect the maximum pressure at the inclusion surface. When the radius of the inclusion is varied there is little change in maximum pressure, at the inclusion's surface, if $R_{1}<<R_{2}$. However in the sensitivity analysis when the radius of the inclusion is about $10 \%$ of the radius of the magma ball the maximum pressures start to vary more rapidly.

The sensitivity analysis for the permeability of the magma shows that if the magma has a low permeability the maximum pressure at the surface of the ejecta is high and this reduces as the permeability increases. Permeability is a measure of how easily fluid flows through a porous rock. At low permeability vapour can not easily escape through the porous magma and this causes the observed high pressure at the surface of the inclusion. The criterion for rupture also shows an increase in the maximum pressure as permeability decreases. Permeability of magma has a wide range of typical values and this results in a wide range of possible maximum pressures.

The sensitivity analysis for both the magma and slurry porosity shows small variations in the maximum pressure at the surface of the inclusion. The criterion for rupture also shows that the maximum pressure at the surface of the inclusion is not affected by the porosity of the magma and slurry. 


\section{Chapter 4}

\section{Conclusion and Discussion}

\subsection{Discussion}

During the derivation of the model for pressure changes in Surtseyan ejecta we assumed:

- That the inclusion would heat uniformly due to the low value of Biot number.

- That the magma ball is much larger than the entrained slurry.

- That the steam generation boundary is fixed.

- That the system is a single phase vapour flow through the magma.

- That the Surtseyan ejecta considered is spherical containing a single inclusion at the centre.

To improve the model these assumptions need to be addressed.

In the thermal equation we considered the inclusion to be heating uniformly and due to the low value of Biot number this is a reasonable assumption. However when heat penetrates into an object the surface of the object heats first and the heat diffuses into the object over time. In order to improve the model a thermal diffusion equation could be used to calculate the temperature. 
In the thermal equation it was also assumed that the magma ball was much larger than the entrained slurry. The result of this is that the heat escaping due to the flow of steam is not considered. When it is assumed that $R_{1}<<R_{2}$ the vapour diffusing through the porous magma has not escaped the magma before the critical time is reached and it can therefore be assumed that no heat escapes. However if we cannot assume that $R_{1}<<R_{2}$ then the heat lost at the interface between the magma and air due to the escaping vapour must be considered.

In the process of solving the model numerically the steam generation boundary is fixed so that the PDEPE function can be used. The justification for this was that the steam generation boundary is much smaller than the radius of the magma ball. This can be assumed because the boundary moves towards the centre of the inclusion from $R_{1}$, which is much smaller than the radius of the magma ball. If the mesh, in the MATLAB code, is updated at each time step towards the origin, we can solve the moving boundary problem on the interval $\left[\frac{s(t)}{R_{2}}, 1\right]$. This produces the numerical solutions seen in Figure 4.1. The maximum pressure observed for a range of parameters is approximately the same as that obtained using a fixed boundary. Therefore the assumption to fix the steam generation boundary did not significantly affect the criteria for rupture.

In the transient model a single phase vapour flow through the magma is assumed. As the water in the inclusion flashes to steam quickly this is a reasonable assumption. If a single phase vapour flow cannot be assumed we need to consider what would happen to the pressure if it were a two phase flow [11]. This would produce a more complex pressure equation which may make the model more accurate.

In the Surtseyan ejecta model we have assumed that a Surtseyan ejecta contains a single slurry inclusion entrained in the centre of the magma. However often Surtseyan ejecta contain many different slurry inclusions scattered in the magma ball. This would create a more complex model as there would be a lack of symmetry. If we were to consider modelling a Surtseyan ejecta with multiple inclusions, we would need to consider how the pressure of each deposit affects the others. Future work modelling Surtseyan ejecta needs to focus on finding a model for this case. 


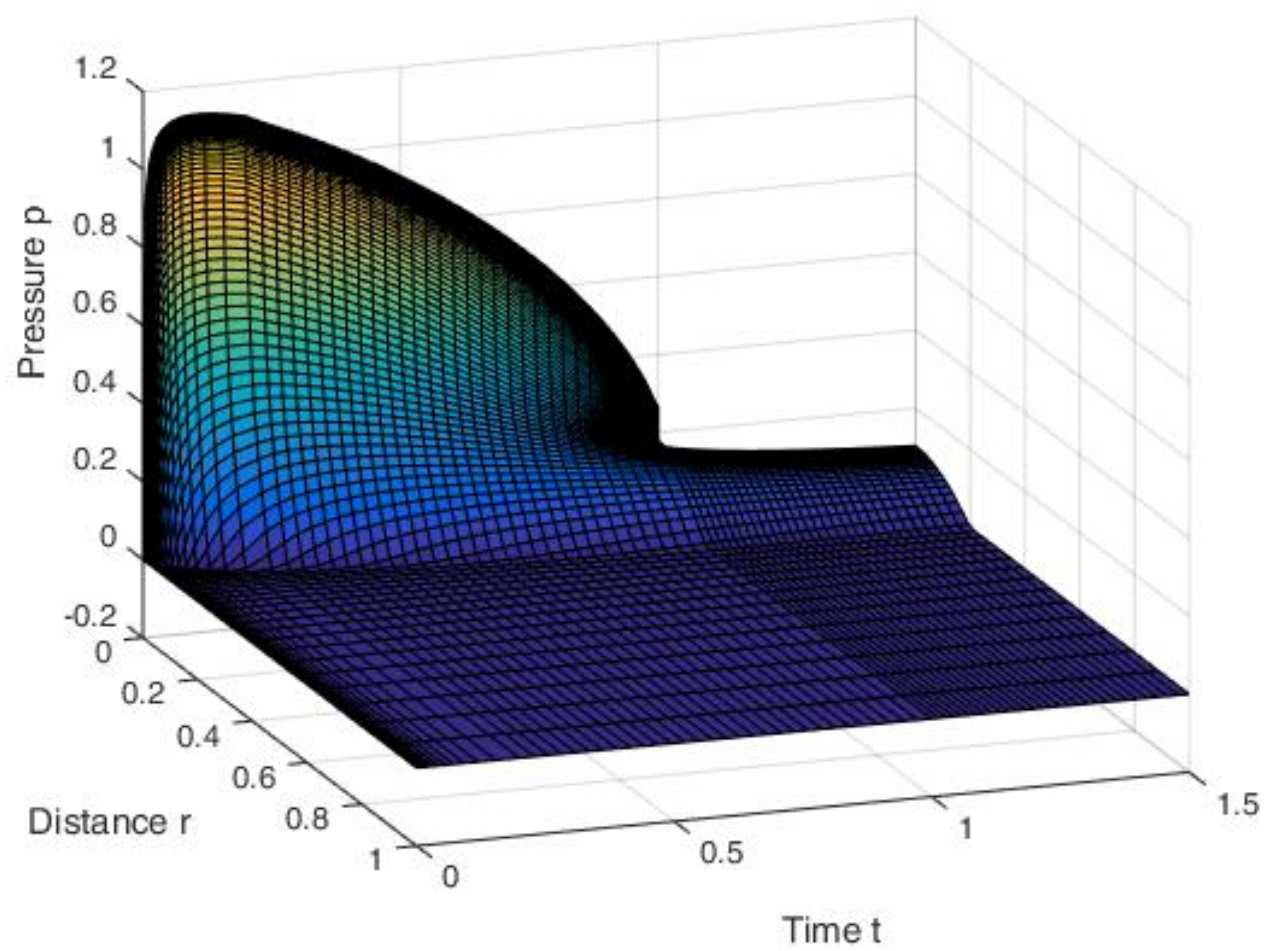

Figure 4.1: Numerical solutions to the non dimensional pressure equations with the parameter as listed in Table 3.2 on a moving mesh that is updated at each time step starting at $\frac{s(t)}{R_{2}}$

\subsection{Conclusion}

In this study we have constructed a mathematical model that allows us to calculate the transient pressure response inside Surtseyan ejecta that only contain one small slurry inclusion situated at the centre of the large magma ball. The pressure increase inside the ejecta is due to the vaporization of the water in the slurry and this is offset by the vapour escaping through the pores of the surrounding magma. Our aim was to determine a criterion for rupture for Surtseyan ejecta.

The numerical solutions of the non dimensional pressure equations showed that the pressure at the surface of the inclusion equilibrated quickly, this occurred for a wide range of parameters. As equilibrium is reach quickly the maximum pressure could be described using steady state equations. The criteria for rupture is that the maximum pressure is greater than 1 . The criterion for rupture was 
calculated to be

$$
\begin{gathered}
\sqrt{\frac{2 R T_{m} \mu_{v} K\left(T_{m}-T_{0}\right)}{M p_{c}^{2} \times 0.28 h_{v l} k}}>1: \text { When } R_{1}<<R_{2}, \\
\sqrt{\frac{2 R T_{m} \mu_{v} K\left(T_{m}-T_{0}\right)}{M p_{c}^{2} \times 0.28 h_{v l} k}\left(\frac{R_{2}-R_{1}}{R_{2}}\right)}>1: \text { When } R_{1}<R_{2} .
\end{gathered}
$$

It was interesting that the criteria for rupture does not depend on the porosity of the inclusion or magma and that it only depends on the size of the radii when $R_{1}$ could not be considered much less than $R_{2}$. As a result it is the permeability and the temperature difference that control when the Surtseyan ejecta will rupture. 


\section{Appendix A}

\section{Symbol Glossary}

\begin{tabular}{|l|l|l|}
\hline \multicolumn{2}{|c|}{ Terms in Chapter 1.2.2 } \\
\hline \multicolumn{2}{|c|}{ Meaning } & Units \\
\hline Symbol & Solid strain tensor & - \\
\hline$e_{i j}$ & Solid displacement & $\mathrm{m}$ \\
$w$ & Fluid displacement & $\mathrm{m}$ \\
$W$ & Yield stress & $\mathrm{Pa}$ \\
$\sigma_{i j} S$ & Elastic constants & \\
$A_{E}, N_{E}, Q_{E}, R_{E}$ & Fluid dilation & $\mathrm{m}$ \\
$\varepsilon_{f}$ & Gas pressure & $\mathrm{Pa}^{-2}$ \\
$p_{g}$ & Porosity & - \\
$\phi$ & Density of fluid & $\mathrm{kg} \mathrm{m}^{-3}$ \\
$\rho_{f}$ & Density of solid & $\mathrm{kg} \mathrm{m}^{-3}$ \\
$\rho_{s}$ & Added mass density & $\mathrm{kg} \mathrm{m}^{-3}$ \\
$\rho_{a}$ & Vertical gas velocity & $\mathrm{m} \mathrm{s}^{-1}$ \\
$v$ & Solid displacement from equilibrium & $\mathrm{m}$ \\
$w_{e}$ & Added mass effect & $\mathrm{kg} \mathrm{m}^{-2} \mathrm{~s}^{-2}$ \\
$A_{m}$ & Interfacial drag & $\mathrm{Pa} \mathrm{m}^{-1}$ \\
$D_{d}$ & Interfacial drag coefficient & $\mathrm{Pa} \mathrm{s} \mathrm{m}^{-2}$ \\
$b$ & Gas viscosity & $\mathrm{Pa} \mathrm{s}^{-2}$ \\
$n_{f}$ & Gas permeability & $\mathrm{m}^{2}$ \\
$k$ & Adiabatic index & - \\
$\gamma$ & Time & $\mathrm{s}$ \\
\hline & & \\
\hline
\end{tabular}




\begin{tabular}{|c|c|c|}
\hline \multicolumn{3}{|c|}{ Terms in Chapter 1.2.3 } \\
\hline Symbol & Meaning & Units \\
\hline$\rho_{x}$ & Density of substance $\mathrm{x}$ & $\mathrm{kg} \mathrm{m}^{-3}$ \\
\hline $\mathbf{u}_{x}$ & Mass flux density of substance $\mathrm{x}$ & $\mathrm{kg} \mathrm{s}^{-1} \mathrm{~m}^{-2}$ \\
\hline$\phi$ & Porosity & - \\
\hline$t$ & Time & $\mathrm{s}$ \\
\hline$\rho_{l}$ & Density of liquid & $\mathrm{kg} \mathrm{m}^{-3}$ \\
\hline$\rho_{v}$ & Density of vapour & $\mathrm{kg} \mathrm{m}^{-3}$ \\
\hline $\mathbf{u}_{l}$ & Mass flux density of liquid & $\mathrm{kg} \mathrm{s}^{-1} \mathrm{~m}^{-2}$ \\
\hline $\mathbf{u}_{v}$ & Mass flux density of vapour & $\mathrm{kg} \mathrm{s}^{-1} \mathrm{~m}^{-2}$ \\
\hline$S_{l}$ & Fraction of pore space containing liquid & - \\
\hline$S_{v}$ & Fraction of pore space containing vapour & - \\
\hline$\rho_{r}$ & Density of rock & $\mathrm{kg} \mathrm{m}^{-3}$ \\
\hline$U_{r}$ & Specific internal energy of rock & $\mathrm{J}$ \\
\hline$U_{l}$ & Specific internal energy of liquid & $\mathbf{J}$ \\
\hline$U_{v}$ & Specific internal energy of vapour & $\mathrm{J}$ \\
\hline$K$ & Thermal conductivity of rock & $\mathrm{W} \mathrm{m}{ }^{-1} \mathrm{~K}^{-1}$ \\
\hline $\mathbf{H}_{l}$ & Specific enthalpy of liquid & $\mathrm{J}$ \\
\hline $\mathbf{H}_{v}$ & Specific enthalpy of vapour & $\mathrm{J}$ \\
\hline \multicolumn{3}{|c|}{ Terms in Chapter 1.2.4 } \\
\hline Symbol & Meaning & Units \\
\hline$F_{x}$ & Mass flux of $x$ & $\mathrm{~kg} \mathrm{~s}^{-1} \mathrm{~m}^{-2}$ \\
\hline$\rho_{v}$ & Density of vapour & $\mathrm{kg} \mathrm{m}^{-3}$ \\
\hline$\phi$ & Porosity & - \\
\hline$t$ & Time & $\mathrm{s}$ \\
\hline$k$ & Absolute permeability or rock & $\mathrm{m}^{2}$ \\
\hline$\mu_{v}$ & Dynamic viscosity of vapour & $\mathrm{m}^{2} \mathrm{~s}^{-1}$ \\
\hline$p$ & Pressure & $\mathrm{Pa}$ \\
\hline $\bar{p}$ & Average pressure & $\mathrm{Pa}$ \\
\hline$p_{i}$ & Initial pressure & $\mathrm{Pa}$ \\
\hline$r$ & Radius & $\mathrm{m}$ \\
\hline$Z$ & Real gas compressibility factor & - \\
\hline$R$ & Gas Constant & $\mathrm{J} \mathrm{K}^{-1} \mathrm{~mol}^{-1}$ \\
\hline$T_{0}$ & Initial temperature of water & $\mathrm{K}$ \\
\hline
\end{tabular}




\begin{tabular}{|c|c|c|}
\hline $\begin{array}{l}M \\
r_{f} \\
q_{v f} \\
H\end{array}$ & $\begin{array}{l}\text { Molecular mass of water } \\
\text { Distance from front to injection site } \\
\text { Mass flow rate at the front } \\
\text { Specific enthalpy }\end{array}$ & $\begin{array}{l}\mathrm{kg} \mathrm{mol}^{-1} \\
\mathrm{~m} \\
\mathrm{~kg} \mathrm{~s}^{-1} \\
\mathrm{~J}\end{array}$ \\
\hline \multicolumn{3}{|c|}{ Terms in Section 2.1} \\
\hline Symbol & Meaning & Units \\
\hline$n_{v}$ & Moles of water vapour & mol \\
\hline$n_{l}$ & Moles of liquid water & mol \\
\hline$m_{l}$ & Mass of liquid water & $\mathrm{kg}$ \\
\hline$M$ & Molecular mass of water & $\mathrm{kg} \mathrm{mol}^{-1}$ \\
\hline$R_{1}$ & Radius of the inclusion & $\mathrm{m}$ \\
\hline$\phi_{r}$ & Porosity of the inclusion & - \\
\hline$\rho_{l}$ & Density of liquid water & $\mathrm{kg} \mathrm{m}^{-3}$ \\
\hline$T(t)$ & Temperature of the inclusion at time $t$ & $\mathrm{~K}$ \\
\hline$T_{m}$ & Temperature of the magma & $\mathrm{K}$ \\
\hline$V$ & Volume of the inclusion & $\mathrm{m}^{3}$ \\
\hline$R$ & Gas Constant & $\mathrm{J} \mathrm{K}^{-1} \mathrm{~mol}^{-1}$ \\
\hline$P$ & Pressure inside the inclusion & $\mathrm{Pa}$ \\
\hline \multicolumn{3}{|c|}{ Terms in Section 2.2} \\
\hline Symbol & Meaning & Units \\
\hline$B_{i}$ & Biot number & - \\
\hline$h$ & Heat Transfer Coefficient & $\mathrm{W} \mathrm{m}^{-1} \mathrm{~K}^{-1}$ \\
\hline$L_{c}$ & Characteristic length of substance $\mathrm{x}$ & $\mathrm{m}$ \\
\hline$V_{c}$ & Volume or substance $\mathrm{x}$ & $\mathrm{m}^{3}$ \\
\hline$A_{c}$ & Area of substance $x$ & $\mathrm{~m}^{2}$ \\
\hline$K_{b}$ & Thermal conductivity of substance $x$ & $\mathrm{~W} \mathrm{~m}{ }^{-1} \mathrm{~K}^{-1}$ \\
\hline$Q$ & Amount of heat & $\mathrm{J}$ \\
\hline$A$ & Area & $\mathrm{m}^{2}$ \\
\hline$\Delta T$ & Change in temperature & $\mathrm{K}$ \\
\hline$\Delta r$ & Change in radius & $\mathrm{m}$ \\
\hline$K_{2}$ & Thermal conductivity of magma & $\mathrm{W} \mathrm{m}^{-1} \mathrm{~K}^{-1}$ \\
\hline$K_{1}$ & Thermal conductivity of inclusion & $\mathrm{W} \mathrm{m}{ }^{-1} \mathrm{~K}^{-1}$ \\
\hline$L_{2}$ & Characteristic length of magma ball & $\mathrm{m}$ \\
\hline$L_{1}$ & Characteristic length of Inclusion & $\mathrm{m}$ \\
\hline
\end{tabular}




\begin{tabular}{|c|c|c|}
\hline $\begin{array}{l}R_{1} \\
R_{2}\end{array}$ & $\begin{array}{l}\text { Radius of the inclusion } \\
\text { Radius of the magma ball }\end{array}$ & $\begin{array}{l}\mathrm{m} \\
\mathrm{m}\end{array}$ \\
\hline \multicolumn{3}{|c|}{ Terms in Section 2.3} \\
\hline Symbol & Meaning & Units \\
\hline$Q$ & Amount of heat & $\mathrm{J}$ \\
\hline$t$ & Time & s \\
\hline$R_{1}$ & Radius of the inclusion & $\mathrm{m}$ \\
\hline$\rho$ & Effective density of the inclusion & $\mathrm{kg} \mathrm{m}^{-3}$ \\
\hline$c_{p}$ & Effective heat capacity at constant pressure & $\mathrm{J} \mathrm{K}^{-1}$ \\
\hline$\rho_{l}$ & Density of liquid water & $\mathrm{kg} \mathrm{m}^{-3}$ \\
\hline$\rho_{m}$ & Density of magma & $\mathrm{kg} \mathrm{m}^{-3}$ \\
\hline$\phi_{r}$ & Porosity of inclusion & - \\
\hline$T$ & Temperature & $\mathrm{K}$ \\
\hline$T_{m}$ & Temperature of magma & $\mathrm{K}$ \\
\hline$T_{0}$ & Initial temperature & $\mathrm{K}$ \\
\hline$r$ & Radius & $\mathrm{r}$ \\
\hline$K_{2}$ & Thermal conductivity of magma & $\mathrm{W} \mathrm{m}{ }^{-1} \mathrm{~K}^{-1}$ \\
\hline$\alpha$ & Thermal equation constant & $\mathrm{kg} \mathrm{s}^{-1}$ \\
\hline \multicolumn{3}{|c|}{ Terms in Section 2.4} \\
\hline Symbol & Meaning & Units \\
\hline$T_{m}$ & Temperature of magma & $\mathrm{K}$ \\
\hline$T_{0}$ & Initial temperature & $\mathrm{K}$ \\
\hline$\phi_{r}$ & Porosity of inclusion & - \\
\hline$\rho_{l}$ & Density of liquid water & $\mathrm{kg} \mathrm{m}^{-3}$ \\
\hline$c_{p}$ & Effective heat capacity at constant pressure & $\mathrm{J} \mathrm{K}^{-1}$ \\
\hline$V$ & Volume of the inclusion & $\mathrm{m}^{3}$ \\
\hline$\Delta T$ & Change in temperature & $\mathrm{K}$ \\
\hline$h_{v l}$ & Latent heat of vaporization of water & $\mathrm{J} \mathrm{kg}^{-1}$ \\
\hline$Q_{1}$ & Energy used to heat water to $373 \mathrm{~K}$ & $\mathrm{~J}$ \\
\hline$Q_{2}$ & Energy used to vaporise water in the ejecta & $\mathbf{J}$ \\
\hline$Q_{3}$ & Energy used to heat water vapour to $T_{m}$ & $\mathrm{~J}$ \\
\hline \multicolumn{3}{|c|}{ Terms in Section 2.5} \\
\hline Symbol & Meaning & Units \\
\hline$T_{m}$ & Temperature of magma & $\mathrm{K}$ \\
\hline
\end{tabular}




\begin{tabular}{|c|c|c|}
\hline$T_{0}$ & Initial temperature & $\mathrm{K}$ \\
\hline$\phi_{r}$ & Porosity of inclusion & - \\
\hline$\rho_{l}$ & Density of liquid water & $\mathrm{kg} \mathrm{m}^{-3}$ \\
\hline$c_{p}$ & Effective heat capacity at constant pressure & $\mathrm{J} \mathrm{K}^{-1}$ \\
\hline$V$ & Volume of the inclusion & $\mathrm{m}^{3}$ \\
\hline$\Delta T$ & Change in temperature & $\mathrm{K}$ \\
\hline$h_{v l}$ & Latent heat of vaporization of water & $\mathrm{J} \mathrm{kg}^{-1}$ \\
\hline$Q_{1}$ & Energy used to heat water to $373 \mathrm{~K}$ & $\mathbf{J}$ \\
\hline$Q_{2}$ & Energy used to vaporise water in the ejecta & $\mathbf{J}$ \\
\hline$Q_{3}$ & Energy used to heat water vapour to $T_{m}$ & $\mathbf{J}$ \\
\hline$Q_{v}$ & Energy used to heat water from $373 \mathrm{~K}$ to $T_{m}$ vapour & $\mathbf{J}$ \\
\hline$V_{m}$ & Volume of magma required to heat the inclusion & $\mathrm{m}^{3}$ \\
\hline$\phi_{m}$ & Porosity of magma & - \\
\hline$R_{1}$ & Radius of the inclusion & $\mathrm{m}$ \\
\hline$R_{\text {used }}$ & Radius of the magma heating the inclusion & $\mathrm{m}$ \\
\hline$\Delta r$ & Change in radius & $\mathrm{m}$ \\
\hline$T$ & Temperature & $\mathrm{K}$ \\
\hline$r$ & Radius & $\mathrm{m}$ \\
\hline$\alpha$ & Thermal equation constant & $\mathrm{kg} \mathrm{s}^{-1}$ \\
\hline \multicolumn{3}{|c|}{ Terms in Section 2.6} \\
\hline Symbol & Meaning & Units \\
\hline$\phi_{m}$ & Porosity of magma & - \\
\hline$\rho_{v}$ & Density of water vapour & $\mathrm{kg} \mathrm{m}^{-3}$ \\
\hline$t$ & Time & s \\
\hline$F_{x}$ & Mass flux of $\mathrm{x}$ & $\mathrm{kg} \mathrm{s}^{-1} \mathrm{~m}^{-2}$ \\
\hline$k$ & Absolute permeability of magma & $\mathrm{m}^{2}$ \\
\hline$\mu_{v}$ & Dynamic viscosity of water vapour & $\mathrm{m}^{2} \mathrm{~s}^{-1}$ \\
\hline$p$ & Partial pressure of water vapour & $\mathrm{Pa}$ \\
\hline$T_{m}$ & Temperature of the magma/lava ball & $\mathrm{K}$ \\
\hline$M$ & Molecular mass of water & $\mathrm{kg} \mathrm{mol}^{-1}$ \\
\hline$R$ & Gas constant & $\mathrm{J} \mathrm{K}^{-1} \mathrm{~mol}^{-1}$ \\
\hline$R_{1}$ & Radius the inclusion & $\mathrm{m}$ \\
\hline$R_{2}$ & Radius of magma/lava ball & $\mathrm{m}$ \\
\hline$r$ & Radius & $\mathrm{m}$ \\
\hline
\end{tabular}




\begin{tabular}{|c|c|c|}
\hline$D$ & Diffusivity constant & $\mathrm{s}$ \\
\hline$s$ & Location of the steam generation boundary & $\mathrm{m}$ \\
\hline$T$ & Temperature & $\mathrm{K}$ \\
\hline$K$ & Thermal conductivity of Inclusion & $\mathrm{W} \mathrm{m}{ }^{-1} \mathrm{~K}^{-1}$ \\
\hline$\rho_{l}$ & Density of liquid water & $\mathrm{kg} \mathrm{m}^{-3}$ \\
\hline$\phi_{r}$ & Porosity of inclusion & - \\
\hline$h_{v l}$ & Latent heat of vaporization of water & $\mathrm{J} \mathrm{kg}^{-1}$ \\
\hline$\Delta t$ & Change in time & s \\
\hline$\dot{s}$ & Velocity of the steam generation boundary & $\mathrm{m} \mathrm{s}^{-1}$ \\
\hline$B$ & Pressure model constant & $\mathrm{m} \mathrm{kg}$ \\
\hline$V_{\text {water }}$ & Volume of water & $\mathrm{m}^{3}$ \\
\hline$m_{\text {vapour }}$ & Volume of water & $\mathrm{m}^{3}$ \\
\hline$t_{c}$ & Critical time & $\mathrm{s}$ \\
\hline$V_{\text {vapour }}$ & Volume of vapour & $\mathrm{m}^{3}$ \\
\hline$\alpha$ & Thermal equation constant & $\mathrm{kg} \mathrm{s}^{-1}$ \\
\hline \multicolumn{3}{|c|}{ Terms in Section 2.7} \\
\hline Symbol & Meaning & Units \\
\hline$\tilde{p}$ & Dimensionless pressure & - \\
\hline$\tilde{r}$ & Dimensionless radius & - \\
\hline$\tilde{t}$ & Dimensionless time & - \\
\hline$\tilde{D}$ & Dimensionless diffusivity constant & - \\
\hline$t_{c}$ & Critical time & $\mathrm{s}$ \\
\hline$p_{c}$ & Critical pressure & $\mathrm{Pa}$ \\
\hline$R_{2}$ & Radius of magma/lava ball & $\mathrm{m}$ \\
\hline$s$ & Location of the steam generation boundary & $\mathrm{m}$ \\
\hline$\dot{s}$ & Velocity of the steam generation boundary & $\mathrm{m} \mathrm{s}^{-1}$ \\
\hline$\alpha$ & Thermal equation constant & $\mathrm{kg} \mathrm{s}^{-1}$ \\
\hline$K$ & Thermal conductivity of Inclusion & $\mathrm{W} \mathrm{m}{ }^{-1} \mathrm{~K}^{-1}$ \\
\hline$\rho_{l}$ & Density of liquid water & $\mathrm{kg} \mathrm{m}^{-3}$ \\
\hline$\phi_{r}$ & Porosity of inclusion & - \\
\hline$M$ & Molecular mass of water & $\mathrm{kg} \mathrm{mol}^{-1}$ \\
\hline$R$ & Gas constant & $\mathrm{J} \mathrm{K}^{-1} \mathrm{~mol}^{-1}$ \\
\hline$R_{1}$ & Radius the inclusion & $\mathrm{m}$ \\
\hline$T_{m}$ & Temperature of the magma/lava ball & $\mathrm{K}$ \\
\hline
\end{tabular}




\begin{tabular}{|c|c|c|}
\hline $\begin{array}{l}k \\
\mu_{v} \\
T_{0} \\
\phi_{m} \\
C\end{array}$ & $\begin{array}{l}\text { Absolute permeability of magma } \\
\text { Dynamic viscosity of water vapour } \\
\text { Initial temperature } \\
\text { Porosity of magma } \\
\text { Dimensionless pressure model constant }\end{array}$ & $\begin{array}{l}\mathrm{m}^{2} \\
\mathrm{~m}^{2} \mathrm{~s}^{-1} \\
\mathrm{~K} \\
- \\
\mathrm{J} \mathrm{s} \mathrm{m}{ }^{-3} \mathrm{~Pa}^{-1}\end{array}$ \\
\hline \multicolumn{3}{|c|}{ Terms in Chapter's 3 and 4} \\
\hline Symbol & Meaning & Units \\
\hline$\tilde{p}$ & Dimensionless pressure & - \\
\hline$\tilde{r}$ & Dimensionless radius & - \\
\hline$\tilde{t}$ & Dimensionless time & - \\
\hline$\tilde{D}$ & Dimensionless diffusivity constant & - \\
\hline$t_{c}$ & Critical time & $\mathrm{s}$ \\
\hline$p_{c}$ & Critical pressure & $\mathrm{Pa}$ \\
\hline$R_{2}$ & Radius of magma/lava ball & $\mathrm{m}$ \\
\hline$s$ & Location of the steam generation boundary & $\mathrm{m}$ \\
\hline$\dot{s}$ & Velocity of the steam generation boundary & $\mathrm{m} \mathrm{s}^{-1}$ \\
\hline$\alpha$ & Thermal equation constant & $\mathrm{kg} \mathrm{s}^{-1}$ \\
\hline$K$ & Thermal conductivity of Inclusion & $\mathrm{W} \mathrm{m}^{-1} \mathrm{~K}^{-1}$ \\
\hline$\rho_{l}$ & Density of liquid water & $\mathrm{kg} \mathrm{m}^{-3}$ \\
\hline$\phi_{r}$ & Porosity of inclusion & - \\
\hline$M$ & Molecular mass of water & $\mathrm{kg} \mathrm{mol}^{-1}$ \\
\hline$R$ & Gas constant & $\mathrm{J} \mathrm{K}^{-1} \mathrm{~mol}^{-1}$ \\
\hline$R_{1}$ & Radius the inclusion & $\mathrm{m}$ \\
\hline$T_{m}$ & Temperature of the magma/lava ball & $\mathrm{K}$ \\
\hline$k$ & Absolute permeability of magma & $\mathrm{m}^{2}$ \\
\hline$\mu_{v}$ & Dynamic viscosity of water vapour & $\mathrm{m}^{2} \mathrm{~s}^{-1}$ \\
\hline$T_{0}$ & Initial temperature & $\mathrm{K}$ \\
\hline$\phi_{m}$ & Porosity of magma & - \\
\hline$C$ & Dimensionless pressure model constant & $\mathrm{J} \mathrm{s} \mathrm{m}^{-3} \mathrm{~Pa}^{-1}$ \\
\hline$\varepsilon$ & Ratio $\frac{R_{1}}{R_{2}}$ & - \\
\hline E & Flux boundary condition constant & $\mathrm{J} \mathrm{m}^{-3} \mathrm{~Pa}^{-1}$ \\
\hline$c_{1}$ & Arbitrary constant 1 & - \\
\hline$c_{2}$ & Arbitrary constant 2 & - \\
\hline$F$ & Pressure criterion constant & - \\
\hline
\end{tabular}




\section{Appendix B}

\section{Programming Numerics in Matlab}

$\% \%$ Simple Pressure Modelling for Surtseyan Ejecta $\% \%$ function SimpleSurtseyanEjectamodel

$\%$ zero partial pressure of water

$\mathrm{pa}=0$;

$\%$ tensile strength of magma

$\mathrm{pc}=2.0 \mathrm{e} 06$;

$\%$ specific heat of vaporisation of water, $\mathrm{J} / \mathrm{kg}$

$\mathrm{Hvl}=2.26 \mathrm{e} 06$;

$\%$ density of liquid water, $\mathrm{kg} / \mathrm{m}^{\wedge} 3$

rhol=999.97;

$\%$ permeability of hardened magma, $\mathrm{m}^{`} 2$

$\mathrm{k}=1.0 \mathrm{e}-14$;

$\%$ thermal conductivity of inclusion, $\mathrm{W} / \mathrm{m} / \mathrm{K}$

$\mathrm{K}=1.2$;

$\%$ dynamic viscosity of vat steam at 10 bar, $\mathrm{Pa} \mathrm{s}$

$\mathrm{mu}=3.0 \mathrm{e}-05$;

$\%$ melting point of magma, in Kelvin

$\mathrm{Tm}=1273$;

$\%$ room temperature, Kelvin

$\mathrm{T} 0=293$;

$\% \mathrm{~T}$ difference driving steam production

$\mathrm{dT}=\mathrm{Tm}-\mathrm{T} 0$; 


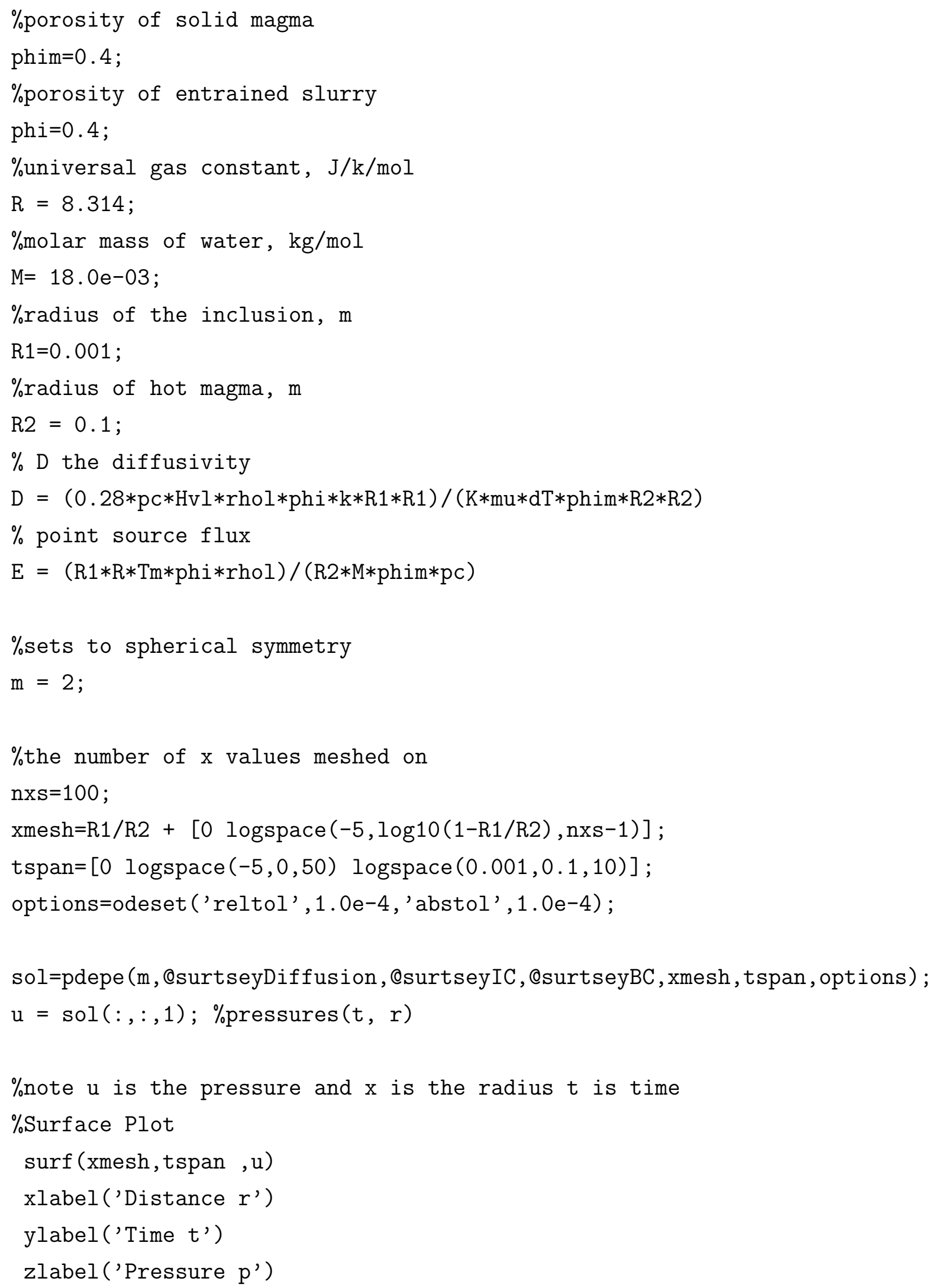




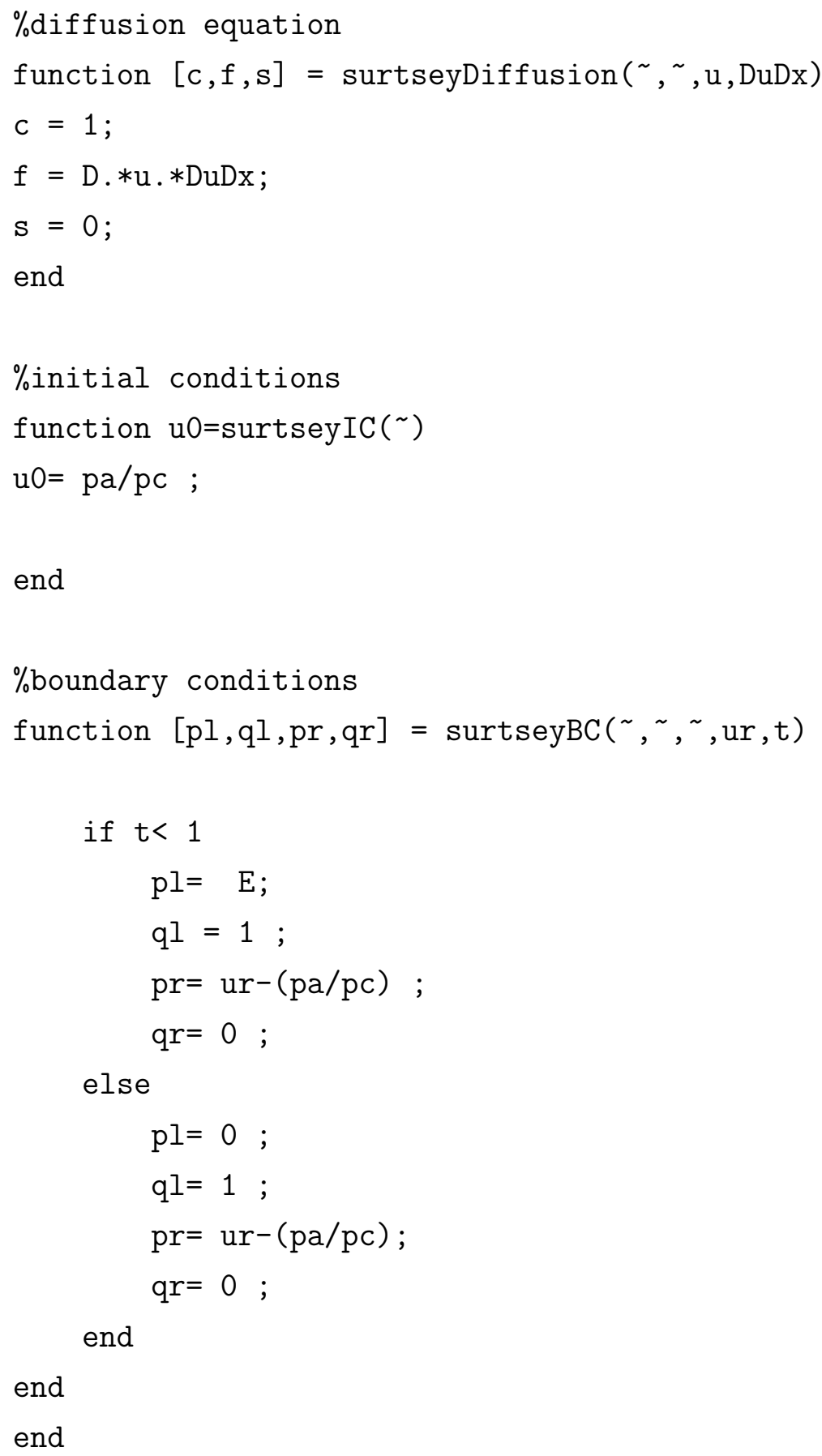




\section{Bibliography}

[1] Alidibrirov, M. A model for viscous magma fragmentation during volcanic blasts. Bulletin of Volcanology 56 (1994), 459-475.

[2] Alidibrirov, M., And Dingwell, D. Three fragmentation mechanisms for highly viscous magma under rapid decompression. Journal of Volcanology and Geothermal Research 100 (2000), 413-422.

[3] Alidibrirov, M., And Panov, V. Magma fragmentation dynamics: experiments with analogue porous low-strength material. Bulletin of Volcanology 59, 7 (1998), 481-490.

[4] Beaton, C. F. Properties of sea water in heat exchanger design handbook. Hemisphere publishing corporation, 1986.

[5] Bennett, F. D. On volcanic ash formation. American Journal of Science 274 (1974), 648-661.

[6] Cengel, Y. A., And Ghajar, A. J. Heat and Mass Transfer: Fundamentals and Applications, 4 ed. McGraw-Hill Science/Engineering/Math, 2011.

[7] Chen, S., Merriman, B., Osher, S., And Smereka, P. A simple level set method for solving stefan problems. Journal of Computational Physics 135, 1 (1997), 8-29.

[8] Colgate, S. A., And Sigurgeirsson, T. Dynamic mixing of water and lava. Nature 244, 5418 (1973), 552-555.

[9] CRAnK, J. The Mathematics of Diffusion. Oxford University Press, 1967. 
[10] Fowler, A. C., Scheu, B., Lee, W. T., And McGuinness, M. J. A theoretical model of the explosive fragmentation of vesicular magma. Proceedings of the Royal Society A: Mathematical, Physical and Engineering Sciences 466, 2115 (2010), 731-752.

[11] Grant, M. A., Donaldson, I. G., And BiXley, P. F. Geothermal Reservoir Engineering. Academic Press, 1982.

[12] ICHIHARA, M. Dynamics of a spherical viscoelastic shell: Implications to a criterion for fragmentation/expansion of bubbly magma. Earth and Planetary Science Letters 265 (2008), 18-32.

[13] KokelaAr, P. The mechanism of Surtseyan volcanism. Journal of the Geological Society 140, 6 (1983), 939-944.

[14] KokelaAr, P. Magma-water interactions in subaqueous and emergent basaltic volcanism. Bulletin of Volcanology 48 (1986), 275-289.

[15] KoKelaAR, P. Discussion of 'Structure and eruptive mechanisms at Surtsey Volcano, Icelend' by J.G. Moore. Geological Magazine (1987).

[16] Mastin, L., And Witter, J. The hazards of eruptions through lakes and seawater. Journal of Volcanology and Geothermal Research 97 (2000), $195-214$.

[17] Mastin, L. G., Christiansen, R. L., Thornber, C., Lowenstern, J., AND BEESON, M. What makes hydromagmatic eruptions violent? Some insights from Keanakāko'i Ash, Kīlauea Volcano, Hawai'i. Journal of Volcanology and Geothermal Reasearch 137 (2004), 15-31.

[18] MCBIRney, A. R. Conductivity variations and terrestrial heat-flow distribution. Journal of Geophysical Research 68, 23 (1963), 6323-6329.

[19] McBirney, A. R., And Murase, T. Factors governing the formation of pyroclastic rocks. Bulletin of Volcanology 34, 2 (1970), 372-384.

[20] Ozisik, M. N. Heat Conduction, 2 ed. Wiley-Interscience Publication, 1993.

[21] Prakash, A., And Mahmood, S. Modified lumped model for transient heat conduction in spherical shape. American International Journal of 
Research in Science, Technology, Engineering and Mathematics 153-176, 153 (2013), 4.

[22] Pruess, K., Calore, C., Celati, R., and Wu, Y. S. An analytical solution for heat transfer at a boiling front moving through a porous medium. Pergmon Journals Ltd. 30, 12 (1987), 2595-2602.

[23] Rohsenow, W. M., AND CHOI, H. Heat, Mass and Momentum Transfer. Prentice-Hall, Inc., 1961.

[24] Singh, H. Dust Eruptions. Master's thesis, Victoria University of Wellington, 2014.

[25] SPARKS, R. S. J. The dynamics of bubble formation and growth in magmas: A review and analysis. Journal of Volcanology and Geothermal Research 3 (1978), 1-37.

[26] Spieler, O., Dingwell, D. B., And Alidibrirov, M. Magma fragmentation speed: an experimental determination. Journal of Volcanology and Geothermal Research 129 (2004), 109-124.

[27] Spieler, O., Kennedy, B., Kueppers, U., Dingwell, D. B., Scheu, B., AND TADDEUCCI, J. The fragmentation threshold of pyroclastic rocks. Earth and Planetary Science Letters 226 (2004), 139-158.

[28] Valentine, G. A. Stratified flow in pyroclastic surges. Bulletin of Volcanology 49 (1987), 616-630.

[29] Verhoogen, J. Mechanics of ash formation. American Journal of Science 249 (1951), 729-739.

[30] White, J. D. L., And Houghton, B. Encyclopedia of Volcanoes. Academic Press, 2000.

[31] Zimanowski, B., Büttner, R., Lorenz, V., And HÄFele, H.-G. Fragmentation of basaltic melt in the course of explosive volcanism. Journal of Geophysical Research 102 (1997), 803-814. 